\title{
IRAK4 mediates colitis-induced tumorigenesis and chemoresistance in colorectal cancer
}

\author{
Qiong Li, ${ }^{1,2}$ Yali Chen, ${ }^{1}$ Daoxiang Zhang, ${ }^{1}$ Julie Grossman, ${ }^{3}$ Lin Li, ${ }^{1}$ Namrata Khurana, ${ }^{1}$ \\ Hongmei Jiang, ${ }^{1}$ Patrick M. Grierson, ${ }^{1}$ John Herndon, ${ }^{1}$ David G. DeNardo, ${ }^{1}$ Grant A. Challen, ${ }^{1}$ \\ Jingxia Liu, ${ }^{4}$ Marianna B. Ruzinova, ${ }^{5}$ Ryan C. Fields, ${ }^{3}$ and Kian-Huat Lim ${ }^{1}$ \\ 'Division of Oncology, Department of Internal Medicine, Barnes-Jewish Hospital and The Alvin J. Siteman \\ Comprehensive Cancer Center, Washington University School of Medicine, St. Louis, Missouri, USA. ²Department of \\ Laboratory Medicine, Renji Hospital, School of Medicine, Shanghai Jiaotong University, Shanghai, China. ${ }^{3}$ Department \\ of Surgery, ${ }^{4}$ Division of Public Health Sciences, Department of Surgery, and ${ }^{5}$ Department of Pathology and Immunology, \\ Barnes-Jewish Hospital and The Alvin J. Siteman Comprehensive Cancer Center, Washington University School of \\ Medicine, St. Louis, Missouri, USA.
}

Aberrant activation of the NF- $\mathrm{kB}$ transcription factors underlies chemoresistance in various cancer types, including colorectal cancer (CRC). Targeting the activating mechanisms, particularly with inhibitors to the upstream IKB kinase (IKK) complex, is a promising strategy to augment the effect of chemotherapy. However, clinical success has been limited, largely because of low specificity and toxicities of tested compounds. In solid cancers, the IKKs are driven predominantly by the Toll-like receptor (TLR)/IL-1 receptor family members, which signal through the IL-1 receptorassociated kinases (IRAKs), with isoform 4 (IRAK4) being the most critical. The pathogenic role and therapeutic value of IRAK4 in CRC have not been investigated. We found that IRAK4 inhibition significantly abrogates colitis-induced neoplasm in $A P C^{\mathrm{Min} /+}$ mice, and bone marrow transplant experiments showed an essential role of IRAK4 in immune cells during neoplastic progression. Chemotherapy significantly enhances IRAK4 and NF- $K B$ activity in CRC cells through upregulating TLR9 expression, which can in turn be suppressed by IRAK4 and IKK inhibitors, suggesting a feedforward pathway that protects CRC cells from chemotherapy. Lastly, increased tumor phosphoIRAK4 staining or IRAK4 mRNA expression is associated with significantly worse survival in CRC patients. Our results support targeting IRAK4 to improve the effects of chemotherapy and outcomes in CRC.

Conflict of interest: The authors have declared that no conflict of interest exists.

Copyright: @ 2019, American Society for Clinical Investigation.

Submitted: June 13, 2019 Accepted: September 4, 2019 Published: October 3, 2019

Reference information: /CI Insight. 2019:4(19):e130867. https://doi.org/10.1172/jici. insight.130867.

\section{Introduction}

The incidence of colorectal cancer (CRC) is rising worldwide $(1,2)$. Systemic chemotherapy is critical in improving survival, but clinical response is highly variable (3). Increased efflux, decreased uptake, and impairment in enzymatic conversion to active metabolites are strategies utilized by CRC cells to lower the effective intracellular concentrations of chemotherapeutics, but these can be partly overcome by combination of different chemotherapeutic agents. However, enhanced survival mechanisms driven by NF-кB, MAPK, $\beta$-catenin, TNF, or PI3K pathways pose an additional therapeutic barrier $(4,5)$. Of these, the NF- $\kappa B$ pathway is one of the best characterized. High basal tumor NF- $\kappa B$ expression is present in at least $40 \%$ of CRC tumor samples and is associated with aggressive phenotype and resistance to chemotherapy (6-9). Genetic ablation of IкB kinase $\beta$-isoform (IKK $\beta$ ), the master kinase within the IKK complex that activates the canonical NF- $\mathrm{KB}$ transcription factors, in intestinal epithelium significantly reduced colitis-induced CRC in mice (10). Besides basal level, NF- $\mathrm{BB}$ activity in CRC cells can be further induced upon exposure to chemotherapy $(7,11)$. Therefore there is strong rationale for targeting the NF- $\mathrm{KB}$ pathway in CRC, particularly through inhibiting the IKK complex (12). Unfortunately, success has been limited to date, as most of the tested inhibitors lack specificity and/ or are highly toxic, as reflected by embryonic lethality of ablation of IKK $\beta$ or RelA in mice (13-16). Therefore, novel, less toxic approaches to target the NF- $\mathrm{KB}$ pathway should be explored. 
In epithelial cells, the IKK/NF- $\mathrm{kB}$ axis can be activated by the Toll-like receptor/IL-1 receptor (TIR) superfamily members, which consist of the Toll-like receptor (TLR) and IL-1 receptor (IL-1R) families (17). Ligation of these receptors results in recruitment and assembly of the adaptor protein MyD88 and IL-1 receptor-associated kinase (IRAK) isoforms 1, 2, and 4, leading to activation of TAK1 kinase and subsequently the IKK complex and NF- $\mathrm{KB}$ (18). Therapeutic value of targeting the IRAK members, specifically the IRAK1 and IRAK4 isoforms, has been elucidated in several cancer types, including diffuse large B cell lymphoma (DLBCL) (19, 20), myelodysplastic syndrome (21), T cell acute lymphoblastic leukemia (22), melanoma (23), breast cancer (24), and head and neck cancer (25), and in pancreatic cancer by our group $(26,27)$. In pancreatic cancer, IL-1 $\beta$ secreted by both neoplastic cells and fibroblasts activates the IRAK4/ $\mathrm{NF}-\mathrm{\kappa B}$ axis to foster tumor fibrosis, which contributes to chemoresistance. Notably, we showed that the kinase activity of IRAK4, but not IRAK1, is essential in driving NF- $\mathrm{BB}$ activity in pancreatic cancer cells (27). Contribution of the TIR receptors and IRAK4 to the progression and treatment resistance of CRC has not been investigated in detail. In an elegant study, $K R A S$-dependent colon cancer cells stimulate BMP-7 secretion to autologously activate BMP receptor, TAK1 kinase, and the NF- $\mathrm{BB}$ transcription factors (28). To date, safe and effective TAK1 inhibitors remain elusive. TAK1 deletion leads to massive bone marrow and liver failure in mice, again raising concerns for toxicities associated with TAK1 inhibitors (29). Contrastingly, an IRAK4 inhibitor (IRAK4i) is currently being tested in a clinical trial (NCT03328078) for refractory DLBCL. In this study, we investigated whether IRAK4 is activated, has a pathogenic role, and is a good therapeutic target in CRC.

\section{Results}

IRAK4 is required for colitis-induced neoplasm in APC $C^{\text {in/+ }}$ mice. We observed robust phosphorylated (denoted p-) IRAK4, p65, and p50 NF- $\mathrm{BB}$ subunit staining by immunohistochemistry (IHC) in colon cancer spontaneously formed in $A P C^{\text {Min/+ }}$ mice, whereas these markers were absent or very faint in normal colon epithelium in age-matched WT littermates (Figure 1A). While $A P C^{\text {Min } /+}$ mice formed almost exclusively small intestinal tumors, treatment with $2 \%$ dextran sodium sulfate (DSS) in drinking water induces colitis and development of colonic neoplasm at very high penetrance (30), and is a robust model for studying colon cancer progression. Using this approach, we found that mice pretreated with DSS followed by an IRAK4i, PF06650833, developed significantly fewer visible tumors and microadenomas compared with vehicle-treated mice, and the number of neoplasms in either sex was similar in both treatment groups (Figure 1, B and C). Intensities of p-IRAK4 and p-p65 IHC staining were drastically diminished in IRAK4i-treated colon, indicating an on-target effect of PF06650833 (Supplemental Figure 1; supplemental material available online with this article; https://doi.org/10.1172/JCI130687DS1). Notably, focused analyses on microadenomas showed that IRAK4i-treated tumors contained significantly fewer proliferating neoplastic (dual $\mathrm{CK}^{+} \mathrm{Ki}-67^{+}$) cells (Figure 1D). Importantly, IRAK4i protected mice from significant weight loss, with no IRAK4i-treated mice reaching humane endpoint while many vehicle-treated mice had to be sacrificed (Figure 1E). To delineate the requirement for IRAK4 in hematopoietic cells in this model, we performed bone marrow transplantation to create $A P C^{M i n /+}$ chimeric mice with IRAK4-null or, as control, WT bone marrow (Figure 2A). Following DSS treatment, $A P C^{\mathrm{Min} /+}$ chimeric mice with IRAK4null bone marrow developed significantly fewer gross tumors and microadenomas compared with mice reconstituted with WT bone marrow, which had numbers of visible tumors and microadenomas similar to those in regular $A P C^{\text {Min/+ }}$ mice (Figure $2 \mathrm{~B}$ ). Notably, mice with transplanted IRAK4-null bone marrow had significantly less colitis, as scored based on crypt damage and inflammatory infiltrates (Figure 2C). These results suggest an essential role for IRAK4 function in hematopoietic cells in colitis-induced neoplastic transformation, reflecting the known role of IRAK4 in innate immunity (31). Intriguingly, we did not observe statistically significant difference in the abundance of $\mathrm{CD}_{4} 5^{+}$leukocytes in the colon epithelium or tumors in either group (Figure 2D), strongly suggesting an essential role for IRAK4 in altering the composition, rather than quantity, of immune infiltrates that mediates colitis and neoplastic progression.

IRAK4 is constitutively activated and drives $N F-\kappa B$ activity in human CRC. We next evaluated activation status of the IRAKs and NF-kB pathway proteins in human CRC. We detected robust p-IRAK1, a direct substrate of IRAK4, in 11 of 12 CRC lines, whereas p-IRAK1 signals were faint in normal colon cell lines FHC and CCD-18Co. On the other hand, p-IRAK4 was detectable at various intensities in both normal and CRC lines (Figure 3A). In these CRC lines, we did not detect an N-terminally truncated, inactive form of IRAK4 protein using an antibody raised against the C-terminus of IRAK4, as reported in myeloid 
A

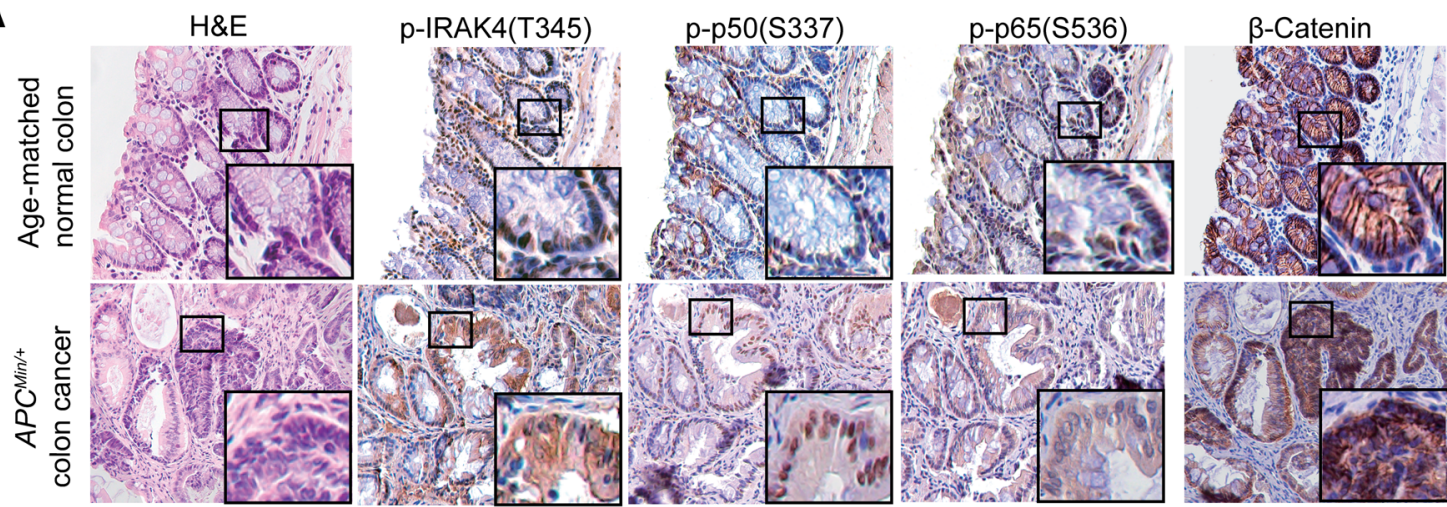

B Treatment scheme
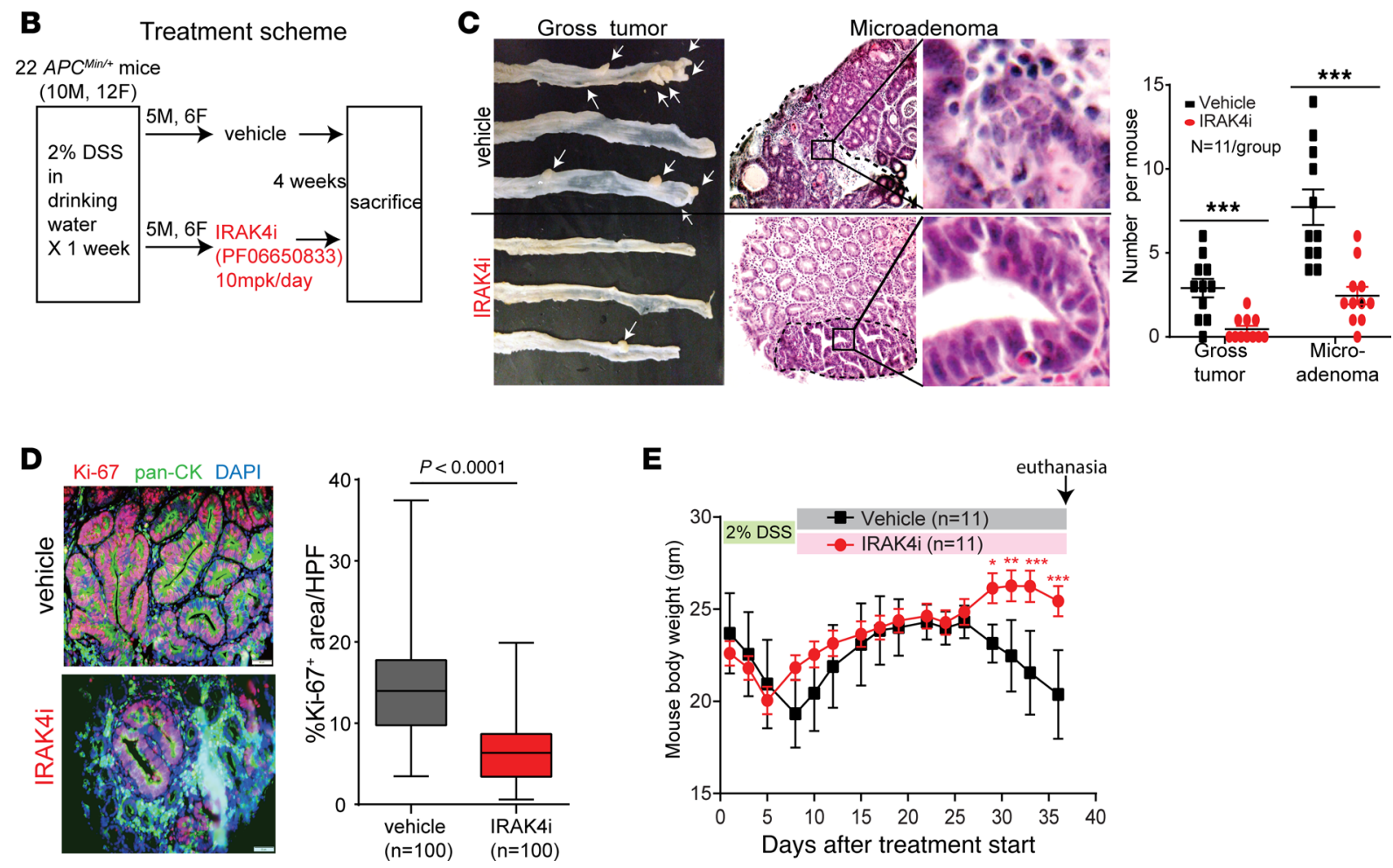

Figure 1. IRAK4 is required for colitis-induced neoplasm in APC $^{\mathrm{Min} /+}$ mice. (A) Representative consecutive H\&E and IHC ( $\left.\times 400\right)$ images of the indicated markers in colon from a 6-month-old [57BL/6) APC ${ }^{\mathrm{Min} /+}$ mouse and WT littermates bred in the same cage. Three pairs of mice were examined showing identical results. (B) Treatment scheme of vehicle or IRAK4i (PF06650833) in APC Min/+ mice after DSS treatment. (C) Representative pictures and quantification of visible colon tumors and microadenomas $(\times 200)$ of treated $A P C^{M i n /+}$ mice (Mann-Whitney test, $\left.{ }^{* * *} P<0.001\right)$. (D) Representative immunofluorescence pictures of dual pan- $\mathrm{CK}^{+}$(green) and $\mathrm{Ki}-67^{+}$(red) cells from colonic neoplasms of $A P C^{\mathrm{Min} /+}$ mice. Quantification of Ki-67+ areas was calculated from 5 random $\times 400$ fields containing pan-CK+ cells of 10 colons per arm (scale bars: $50 \mu \mathrm{m} ; 2$-tailed $t$ test). (E) Serial measurements of body weight of $A P C^{\text {Min } /+}$ mice treated as indicated. Data are presented as means \pm SEM (ANOVA, ${ }^{*} P<0.05,{ }^{* *} P<0.01,{ }^{* *} P<0.001$ ).

malignancies (ref. 32 and Supplemental Figure 2A). Notably, p-IKK $\alpha / \beta, \mathrm{p}$-p65, and p-p50 were detected predominantly in CRC lines. In this limited panel of cell lines, we did not observe any correlation between known genetic mutations ( $K R A S, P I K 3 C A$, and $B R A F$ ) and activation status of these NF-kB pathway molecules, indicating that NF-KB activity cannot be attributed to these oncogenic events. Next, we performed IHC analyses using a pool of 5 commercial human CRC tissue microarrays (TMAs). We found p-IRAK4 IHC staining to be significantly stronger in CRC $(n=220)$ compared with normal colon tissues $(n=49$; Figure $3 \mathrm{~B}$ ), although a fraction of normal colon mucosa also stained robustly with p-IRAK4. The staining intensity of p-IRAK4 did not differ among CRC from various clinical stages (Supplemental Figure 2B). Perhaps relevantly, expression of IRAK4 mRNA is significantly higher in colon cancer than in normal colon tissues from analysis of Oncomine (33), a public microarray database (Supplemental Figure 2C). 
A

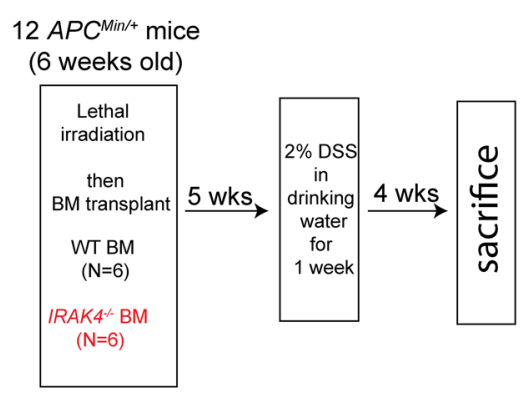

C

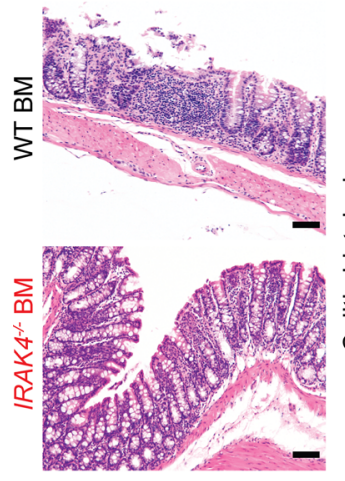

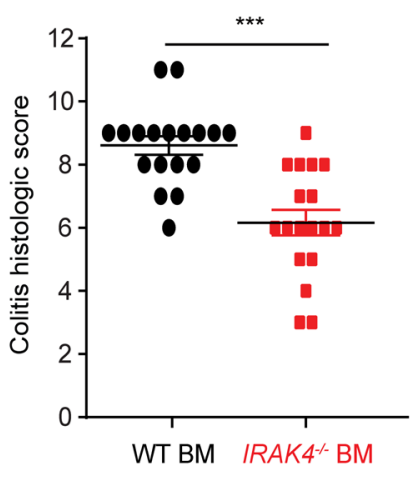

B

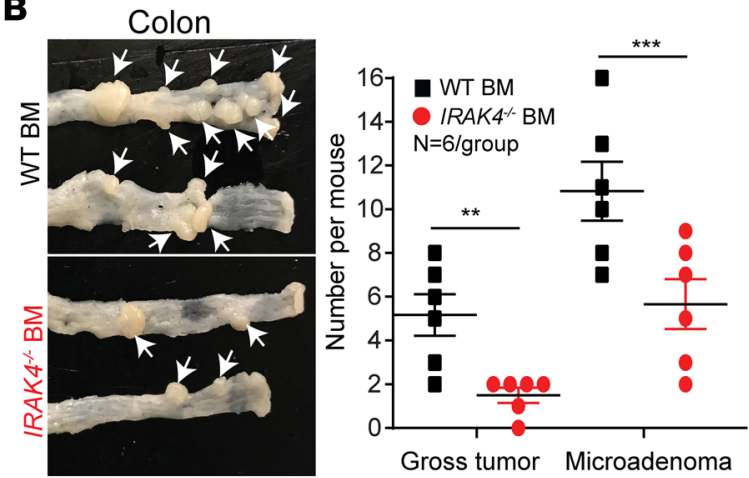

D

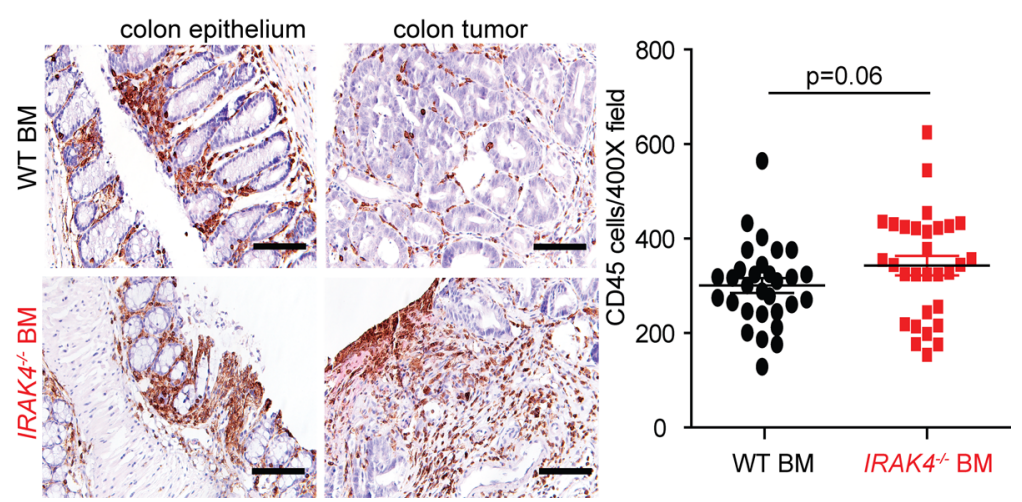

Figure 2. Bone marrow IRAK4 is required for colitis-induced neoplasm in APC ${ }^{\mathrm{Min} /+}$ mice. (A) Treatment scheme of $A P C^{\mathrm{Min} /+}$ mice. (B) Representative pictures and quantification of visible colon tumors and microadenomas from DSS-treated APC ${ }^{\mathrm{Min} /+}$ mice pretransplanted with WT or IRAK4-null bone marrow (Mann-Whitney test, ${ }^{* *} P<0.01,{ }^{* *} P<0.001$ ). (C) Representative IHC pictures and quantification of degree of colitis of colonic tissues from DSS-treated $A P C^{\mathrm{Min} /+}$ mice pretransplanted with WT or IRAK4-null bone marrow. For each group of 6 colons, 3 random $\times 400$ fields were scored and presented as mean $\pm \mathrm{SEM}$ (2-tailed $t$ test, ${ }^{* *} P<0.001$ ). (D) Representative IHC pictures and quantification of CD45+ cells from colon of DSS-treated chimeric $A P C^{\text {Min/ } /}$ mice. For each group, 5-6 random $\times 400$ pictures were taken and $C D 45^{+}$cells counted using Image) software; data are presented as mean \pm SEM (2-tailed $t$ test). Scale bars: $50 \mu$ m.

Upregulated p-IRAK4 staining was also present in benign colonic polyps, although the number of samples was low $(n=10)$. Interestingly, p-IRAK4 staining was higher in tumor-adjacent normal colon samples compared with noncancerous colon samples. These results suggest that IRAK4 can be activated as early as the precancerous stage and participates in neoplastic progression, echoing our observation in $A P C^{\text {Min/+ }}$ mice. Notably, we observed strong, significant correlation between the p-IRAK4 and the p-p65 IHC staining in colon cancer samples (Figure 3C). We did not use p-p50 for correlation because the distribution of p-p50 is almost exclusively nuclear, precluding accurate interpretation of staining intensity. Together, these results establish a strong correlation between activation of IRAK4 and activation of the NF- $\mathrm{KB}$ pathway during CRC progression.

IRAK4 drives $N F-\kappa B$ activity in human and murine CRC cells. To investigate the causal relationship between IRAK4 and NF- $\mathrm{KB}$ activity, we stably silenced IRAK4 in 2 CRC lines, DLD-1 and KM12, using 2 different small hairpin RNAs (shRNAs). We observed decreased p-IRAK1, p-IKK $\alpha / \beta$, p-p50, p-65, and nuclear p65 levels in IRAK4-silenced cells (Figure 4, A and B). NF- $\mathrm{kB}$ activity in IRAK4-silenced cells can be rescued by reexpression of WT IKK $\beta$ or, more potently, activated mutant of IKK $\beta$ (Supplemental Figure 3A), placing IRAK4 as the upstream event of NF- $\mathrm{KB}$ activity. Notably, IRAK4-silenced CRC cells were also defective in anchorage-independent growth and tumorigenesis (Figure 4, C and D). To complement our findings in a murine model, we also investigated the cell-autonomous role of IRAK4 in MC38 cells, a murine CRC line derived from a C57BL/6J mouse (34). Using CRISPR/Cas9 technique, we generated polyclonal MC38 lines stably expressing Cas9 and 2 different IRAK4-targeting sgRNAs. Compared with a vector control line, IRAK4-ablated MC38 lines were severely defective in clonogenic growth, anchorage-independent growth, and tumorigenesis (Figure 4, E-F). As a result, mice bearing IRAK4-ablated MC38 tumors survived significantly longer than those bearing scramble control tumors (Figure 4G). Notably, IRAK4 sgRNA-transduced 
A

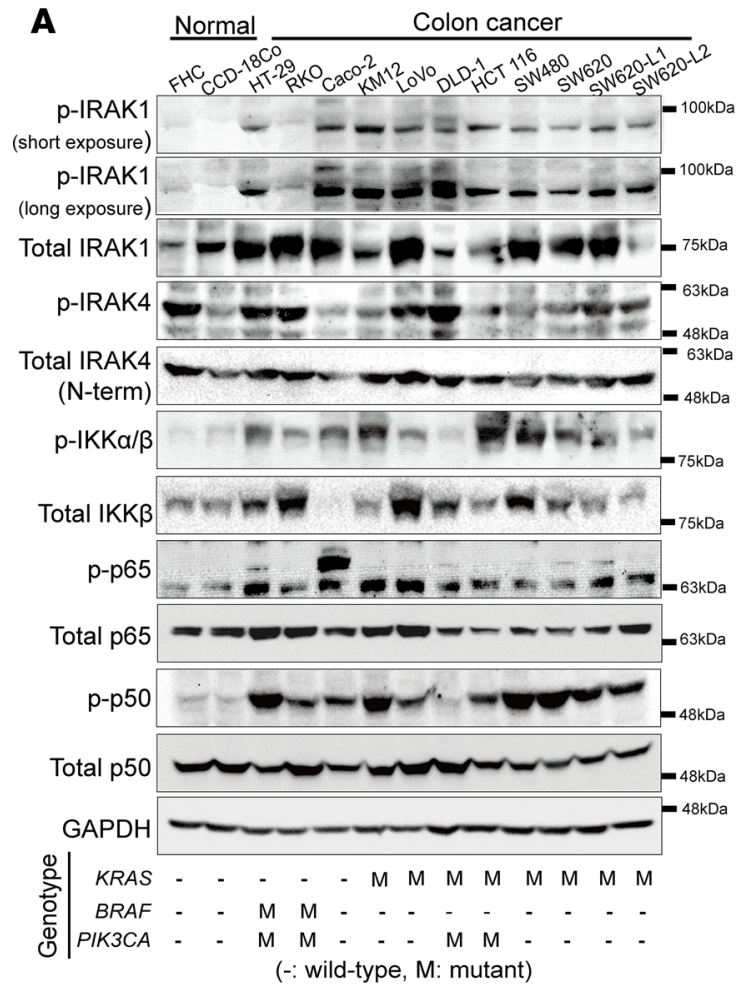

B
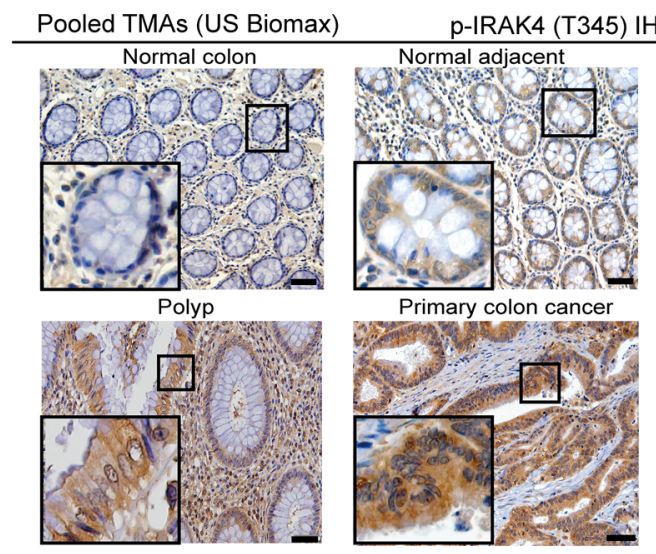

Primary colon cancer
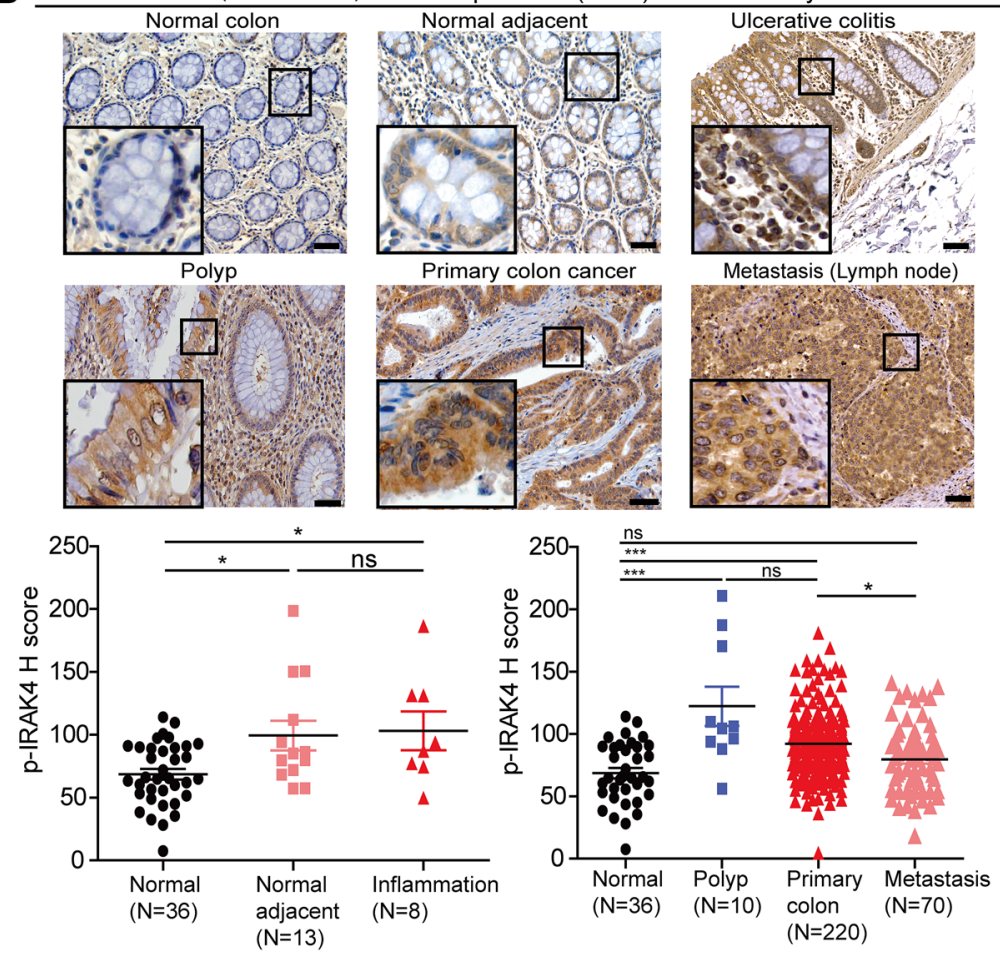

C IHC score (manually scored by GI pathologist)
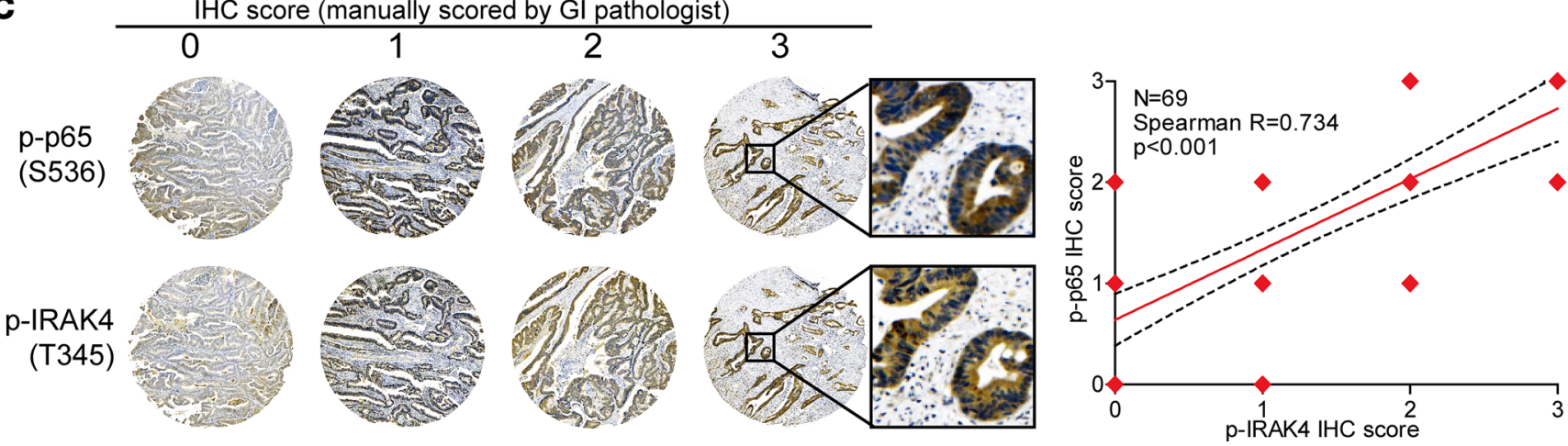

Figure 3. IRAK4 is constitutively activated in human CRC. (A) Western blots showing phosphorylation (denoted p-) status of IRAK1, IRAK4, IKK $\alpha / \beta$, p65, and p50 in normal colon versus colon cancer cell lines with different oncogenic background. (B) Representative p-IRAK4 IHC images and H scores based on epithelial compartment of normal colon, normal colon tissues adjacent to cancer, polyp, and colon cancer pooled from 5 different commercial TMAs (ANOVA, ${ }^{*} P<0.05,{ }^{* * *} P<0.001$; scale bars: $50 \mu \mathrm{m}$ ). (C) Representative $\mathrm{p}$-IRAK4 IHC images and assigned scores of $p$-IRAK4 and $p$-p65 IHC staining on colon cancer samples on a commercial TMA (US Biomax CO7O2a). Staining intensity and scoring were interpreted by gastrointestinal pathologist MBR and Spearman correlation between intensities of both markers analyzed using GraphPad Prism 6.0.

MC38 tumors eventually grew but, when recultured, were all found to have restored IRAK4 expression (Supplemental Figure 3B). We conclude that IRAK4 is necessary for both the hematopoietic and epithelial compartments in CRC development and progression. Together, our results strongly support that IRAK4 is the driver of the canonical NF- $\mathrm{KB}$ pathway and is required for tumorigenesis of CRC cells.

IRAK4 inhibitors suppress NF- $\kappa B$ activity in CRC cells. To translate our findings, we tested 2 different IRAK4 inhibitors. Of these, PF06650833 is being tested in clinical trial (35), and AS2444697 was shown to protect inflammation-associated renal failure in rats (36). We previously showed that AS2444697 blocks IL-1 $\beta$-induced NF- $\mathrm{KB}$ activation in pancreatic cancer cells $(27,36)$. Here, we observe varied susceptibility of different CRC lines to both IRAK4 inhibitors in standard 2-dimensional cultures (Figure 5A). Importantly, we observed strong positive correlation in $\mathrm{IC}_{50}$ values between the two IRAK4 inhibitors across 13 
A

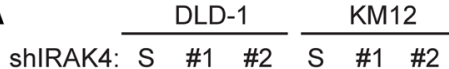
Total IRAK4 - - - - - - $48 \mathrm{kDa}$ $\mathrm{p}-\mathrm{IRAK} 1=-\ldots-100 \mathrm{kDa}$ Total IRAK1 $=-75 \mathrm{kDa}$ $\mathrm{p}-\mathrm{IKKa} / \mathrm{\beta} \longrightarrow-75 \mathrm{kDa}$ Total IKKB $\square-75 \mathrm{kDa}$ p-p50

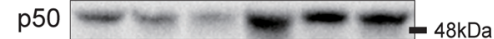
p-p65 $=63 \mathrm{kDa}$ p65 GAPDH

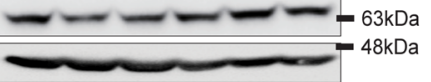

D

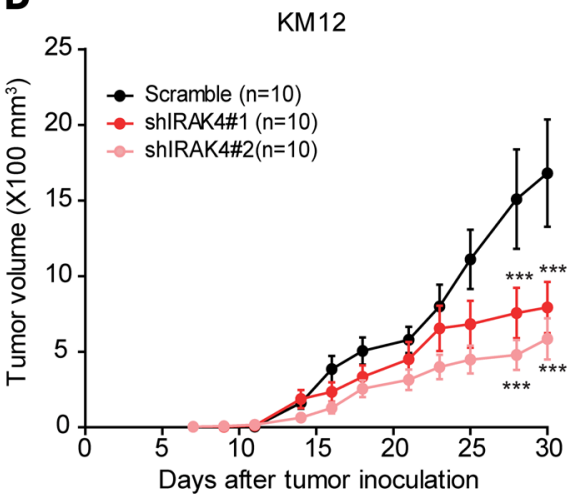

$\mathbf{F}$ $\mathrm{MC38}$

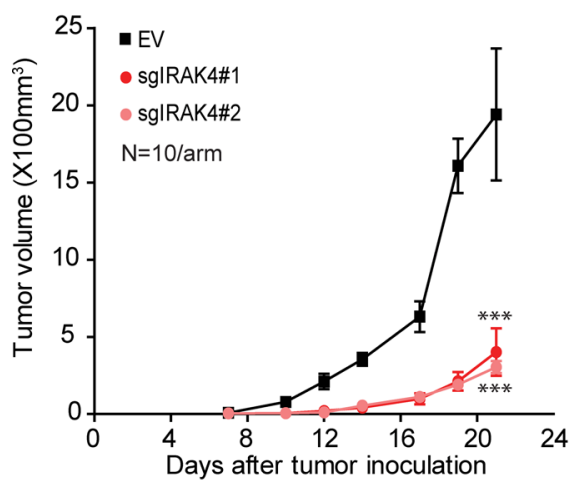

B

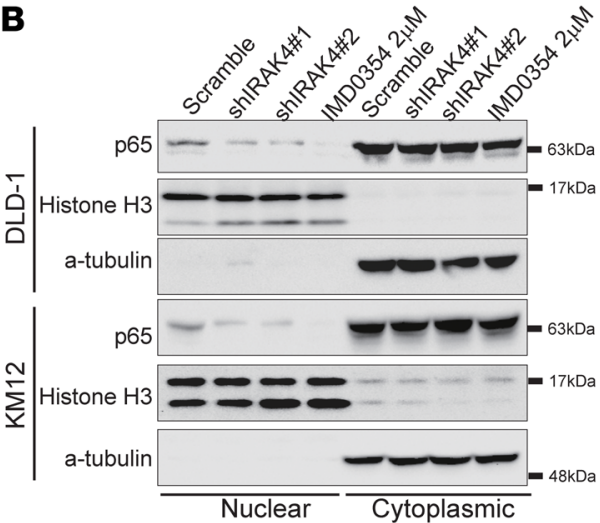

E

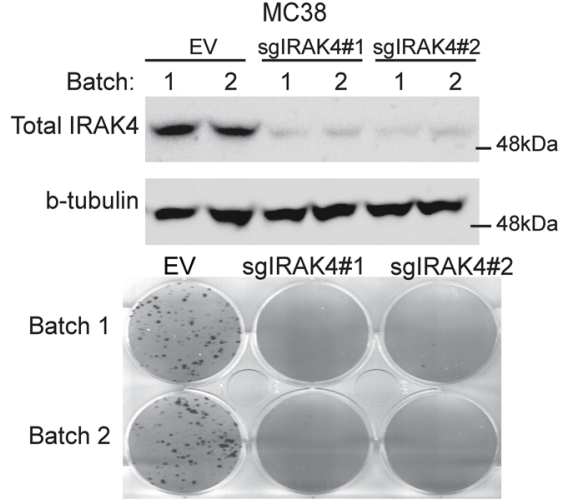

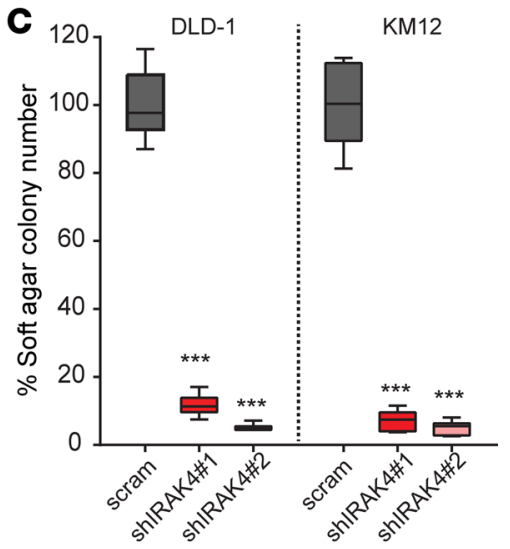

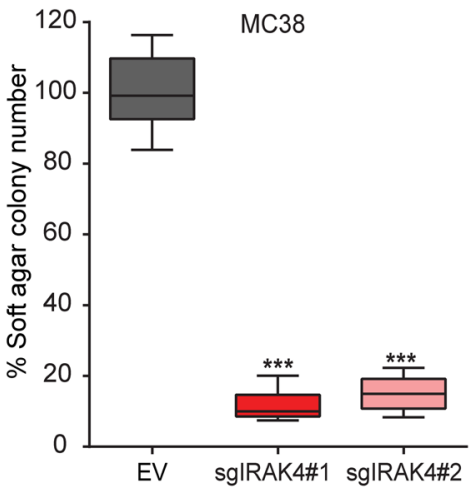

G

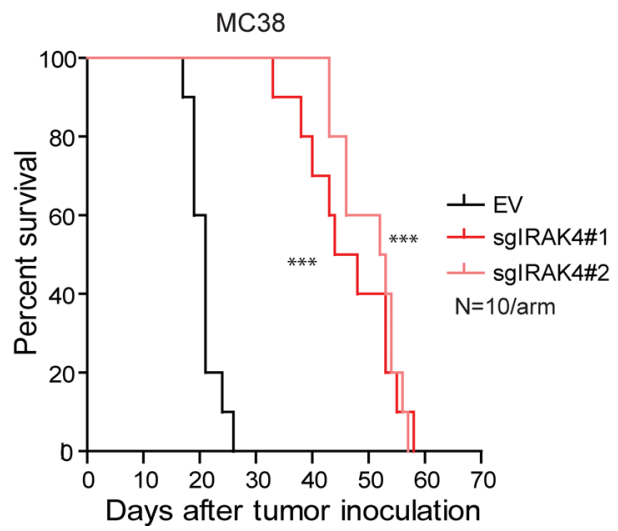

Figure 4. IRAK4 drives NF-кB activity in human and murine CRC cells. (A) Western blots showing the effect of stable IRAK4 knockdown on the indicated markers, compared with scramble (S) control cells. (B) Western blots showing diminished nuclear p65 following IRAK4 knockdown in 2 CRC lines. Treatment with the IKK $\beta$ inhibitor IMD-0354 overnight served as control. (C) Relative quantification of soft agar colonies formed by DLD-1 and KM12 cells stably expressing scramble or 2 IRAK4 shRNAs grown over 3 weeks. Data represent 1 of 3 sets of experiments each done in triplicate and presented as mean \pm SEM (ANOVA, ${ }^{* *} P<0.001$ ). (D) Tumor kinetics of the indicated KM12 tumors grown subcutaneously in bilateral flanks of nude mice ( $n=5$ mice or 10 tumors per group). Data represent mean tumor volume \pm SEM (ANOVA, $\left.{ }^{* * *} P<0.001\right)$. (E) Western blots showing IRAK4 protein levels in MC38 cells infected with pLentiCRISPR/Cas9 alone or with 2 sgRNAs targeting IRAK4. Two different polyclonal batches of cells were made and pooled for colony formation assay (14 days) and soft agar assay (21 days). Data represent 1 of 3 sets of soft agar experiments each done in triplicate and presented as mean \pm SEM (ANOVA, $\left.{ }^{* * *} P<0.001\right)$. (F) Growth kinetics of the indicated MC38 cells grown in C57BL/6] mice (ANOVA, ${ }^{* * *} P<$ 0.001). (G) Kaplan-Meier survival analysis of the indicated MC38 cells grown in C57BL/6] mice (log-rank, $\left.{ }^{* * *} P<0.001\right)$.

CRC lines, cross-validating the specificity of these two compounds (Figure 5B). Supporting our results using shRNAs, both IRAK4 inhibitors suppressed clonogenic growth of CRC cells (Figure 5C). Notably, while IRAK4 inhibitors did not suppress growth of SW80 and SW620 cells in monolayer culture, they potently suppressed clonogenic growth of these cells, implying engagement and need for IRAK4 and $\mathrm{NF}-\kappa \mathrm{B}$ activation in stress-induced conditions, as we previously reported in pancreatic cancer cells (26). 
Both IRAK4 inhibitors potently blocked p-IRAK1, p-p50, nuclear translocation of p65, and NF-kB-driven luciferase reporter activity dose-dependently in CRC cells (Figure 5, D-F). Notably, PF06650833 was also able to dose-dependently suppress p-IRAK4 and p-IRAK1 in normal colon cell line FHC (Supplemental Figure 3C), suggesting IRAK4 as the upstream activator of IRAK1 in non-neoplastic colon cells. As further validation of their specificity, the inhibitory effects of PF06650833 and AS2444697 on NF- $\mathrm{KB}$ reporter can be completely abolished by ectopic expression of constitutively active IKK $\beta$ mutant (37), but not WT IKK $\beta$ (Figure 5G). These results validate the utility of these IRAK4 inhibitors as NF- $\mathrm{BB}$ suppressors in CRC.

Chemotherapy induces TLR9-IRAK4 circuitry to sustain CRC cell survival. Besides basal activity, DNA damage such as that induced by chemotherapy can activate ATM, which can bind and activate TAK1 and IKK $\gamma$ (NEMO) subunit, resulting in further activation of the canonical NF- $\mathrm{kB}$ cascade, which allows cells to survive and undergo DNA repair (38). Therefore, abrogating NF- $\kappa B$ induction represents a promising strategy to potentiate the cytotoxic effect of chemotherapy (39). As expected, we found that exposure of CRC cells to chemotherapy significantly upregulated $\mathrm{NF}-\mathrm{kB}$ reporter activity in CRC cells (Figure 6A). While oxaliplatin or 5-FU alone was able to modestly activate $\mathrm{NF}-\mathrm{kB}$ activity, the combination of both agents led to a more significant upregulation, indicating that the degree of NF- $\mathrm{KB}$ induction is not specific to any class of chemotherapeutic but is more likely associated with the extent of DNA damage. To explore whether IRAK4 is required for chemotherapy-induced NF- $\mathrm{KB}$ activation, we tested CRC cells with oxaliplatin or 5-FU separately in the absence or presence of PF06650833 or AS2444697. Both IRAK4 inhibitors significantly suppressed NF-kB activity and enhanced apoptosis of CRC cells treated with either chemotherapy agent, indicating that the suppressive effect of IRAK4i is not specific to any chemotherapeutic agent (Supplemental Figure 4, A-C). Similarly, IRAK4-silenced CRC cells were significantly more sensitive to 5-FU, oxaliplatin, and SN-38 (a metabolite of irinotecan; Supplemental Figure 4D). Because IRAK4 is typically activated by TLRs or IL-1R, we examined expression levels of these receptors as well as $\mathrm{IL}-1 \alpha$ and $\mathrm{IL}-1 \beta$ following chemotherapy (5-FU plus oxaliplatin) exposure. Of these receptors, TLR9 was the most significantly upregulated in all $3 \mathrm{CRC}$ lines following exposure to chemotherapy (Figure 6B). Notably, 5-FU and, to a lesser degree, oxaliplatin were able to induce TLR9 expression and p-IRAK4, but the combination of both agents was at least additive (Figure 6C, left panel). Notably, chemotherapy-induced TLR 9 coincided with upregulated p-IRAK4 and $p$-IKK $\alpha / \beta$ (Figure $6 \mathrm{C}$, right panel). Because TLR9 signals through IRAK4 and IKK (40), we postulate that TLR9 may play a role in chemotherapy-induced NF- $\mathrm{BB}$ activation. Supporting this notion, we observed significantly increased interaction between TLR9 and p-IRAK4 in chemotherapy-treated CRC cells by proximal ligation assay using TLR9 and p-IRAK4 antibodies (Figure 6D). Interestingly, some level of interaction was already present in control cells, indicating TLR9 as an activator of IRAK4 and NF- $\mathrm{KB}$ at baseline. Knockdown of TLR9 by 2 different shRNAs increased apoptosis, as determined by PARP cleavage, and completely abrogated chemotherapy-induced NF- $\mathrm{BB}$ activation (Figure 6, E and F). Conversely, IRAK4i or IKK $\beta$ inhibitor (IMD-0354) partially suppressed chemotherapy-induced TLR9 mRNA and protein expression and promoted apoptosis (Figure 6, G and $\mathrm{H}$ ). These results suggest that TLR9 is a transcriptional target of NF- $\mathrm{kB}$ during DNA damage. Intriguingly, chemotherapy also enhanced cleavage of TLR9, a marker for TLR9 activation, in 293T cells (Supplemental Figure 5). Because TLR9 is known to sense double-stranded DNAs, which presumably are enriched following DNA damage, these data depict a scenario where damaged DNA may bind and activate TLR9, leading to IRAK4-IKK-NF-kB activation, which in turn further upregulates TLR9 expression, forming a feed-forward circuitry to sustain cellular survival. Therefore, breaking this circuitry with IRAK4i or IMD-0354 can enhance the proapoptotic effect of chemotherapy. Together, these results provide strong rationale for testing IRAK4 inhibitors in combination with chemotherapy in vivo.

IRAK4 inhibition synergizes with chemotherapy in vitro and in vivo. We next quantified the drug effect interactions between the 2 IRAK4 inhibitors and oxaliplatin, SN-38, and 5-FU across 6 fixed-ratio doses in 3 CRC cell lines, using the widely adopted Chou-Talalay method (CompuSyn software, ComboSyn) (41). Of the 3 CRC lines tested, most combination index values fell below 0.9, the defined cutoff for synergism (ref. 41 and Figure 7, A and B). Next, we investigated whether IRAK4i will potentiate chemotherapy in xenograft mouse models. We chose to focus on oxaliplatin and 5-FU because these 2 agents (in the form of FOLFOX) are the most common first-line agents administered in advanced CRC and in the postoperative setting. To this end, we focused on DLD-1 and KM12 cells, which readily form tumors in immunocompromised mice with very short latency ( $\sim 14$ days). Established $\left(\sim 100 \mathrm{~mm}^{3}\right)$ subcutaneous KM12 and DLD-1 tumors growing in nude mice were treated. Although neither AS2444697 nor chemotherapy alone was able to significantly suppress tumor growth, in both models combination-treated tumors were significantly slower in growth kinetics and volume (Figure 7, C and G). Combination-treated tumors showed significantly larger areas of necrosis, more 
A

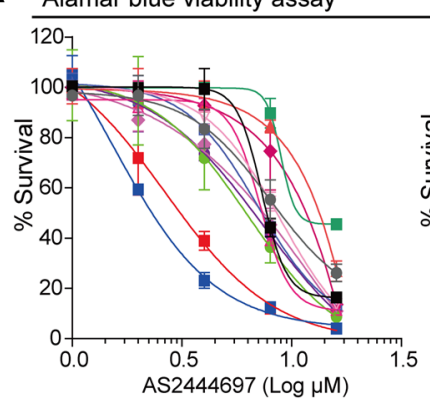

C

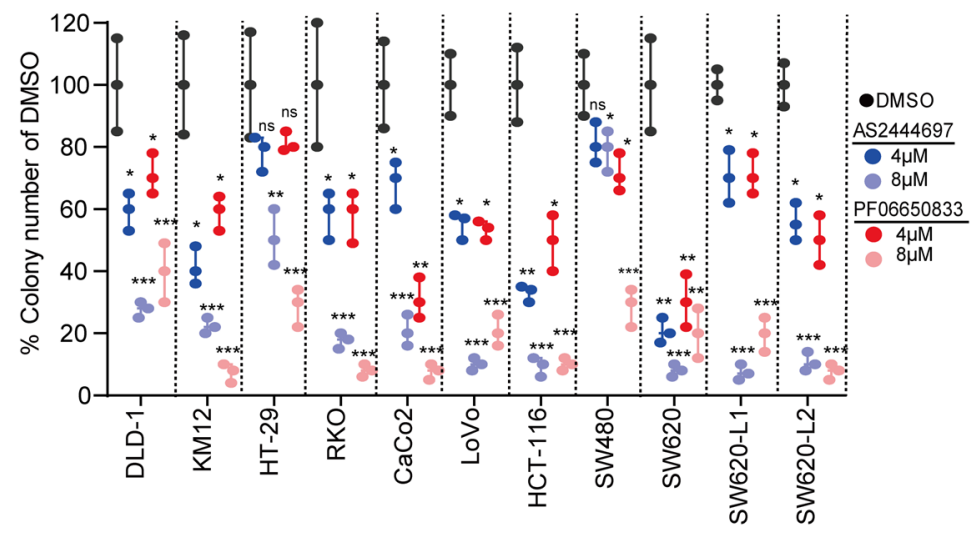

B

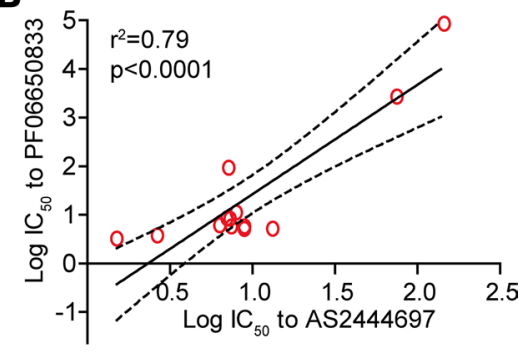

E

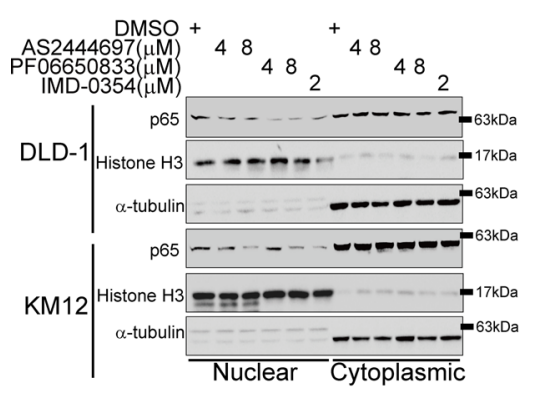

$\mathbf{F}$

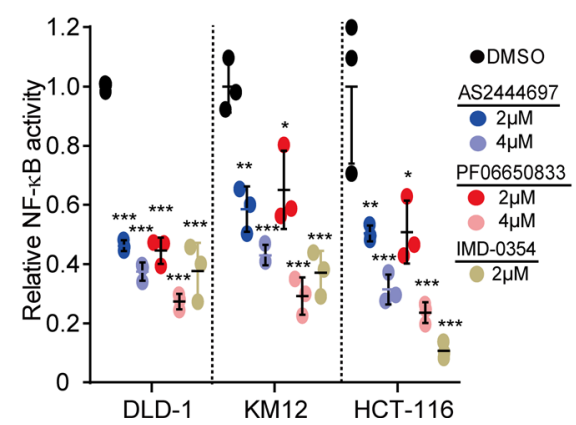

D

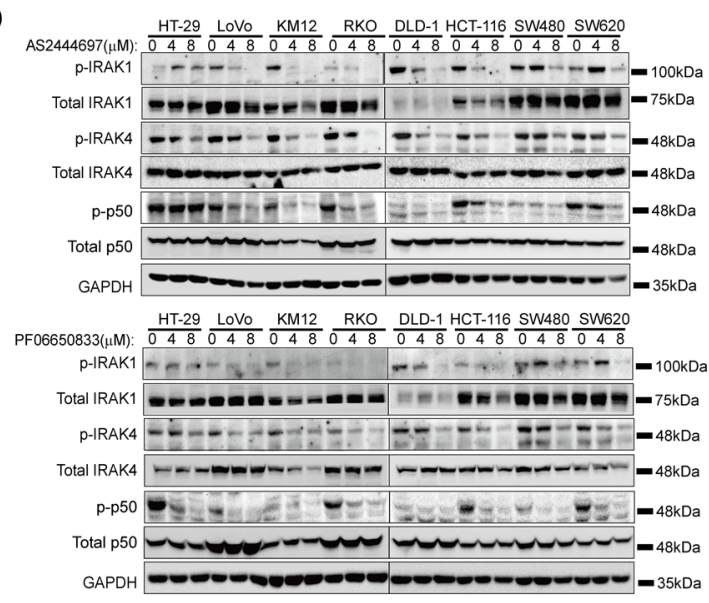

G

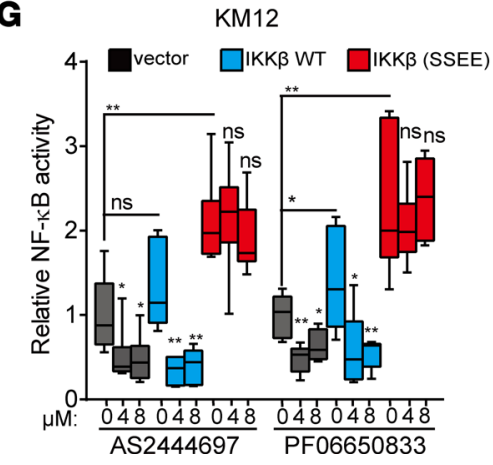

Figure 5. IRAK4 inhibitors suppress NF-KB activity in CRC cells. (A) Alamar Blue assay showing the viability of colon cancer lines cultured in serial concentrations of 2 different IRAK4 inhibitors for 5 days, and the respective $I_{50}$ values. Data represent 1 of 3 sets of experiments each done in triplicate. (B) Correlative analyses of $I_{50}$ values between the 2 indicated IRAK4 inhibitors in colon cancer cell lines. (C) Quantification of clones formed by the indicated CRC lines treated with DMSO or 2 different IRAK4 inhibitors over 3 weeks. Data represent 1 of 3 sets of experiments each done in triplicate and presented as mean \pm SEM (ANOVA, ${ }^{*} P<0.05,{ }^{* *} P<0.01,{ }^{* * *} P<0.001$ ). (D) Western blots showing the suppressive effect of 2 different IRAK4 inhibitors on the indicated markers after overnight treatment. (E) Western blots showing diminished abundance of nuclear $\mathrm{p} 65$ in 2 different CRC cell lines treated with both IRAK4 inhibitors overnight. The IKK $\beta$ inhibitor IMD-0354 served as positive control. (F) NF-KB luciferase reporter assay of different CRC lines incubated with DMSO or the indicated inhibitors overnight. Data represent 1 of 3 sets of experiments each done in triplicate and presented as mean \pm SEM (ANOVA, ${ }^{*} P<0.05,{ }^{* *} P<0.01,{ }^{* *} P<0.001$ ). (G) NF- $\mathrm{kB}$ luciferase reporter assay of KM12 cells transfected with empty vector, WT, or constitutively activated (S177E/S181E) IKK $\beta$ incubated with DMSO or the indicated IRAK4 inhibitors overnight. Data represent 1 of 3 sets of experiments each done in triplicate and presented as mean \pm SEM (Tukey's multiple-comparisons test, ${ }^{*} P<0.05,{ }^{* *} P<0.01$ ).

apoptosis (by cleaved caspase- $3^{+}$area), and less proliferation $\left(\mathrm{Ki}-67^{+}\right)$of neoplastic cells (dual pan-CK $\mathrm{Ki}^{+} 67^{+}$ cells) compared with the other treatment arms (Figure 7, D, E, H, and I). Notably, p-IRAK4 and p-p50 IHC staining was reduced in AS2444697-treated tumors, confirming on-target effects (Supplemental Figure 6A). To further confirm and potentially translate our findings, we repeated the experiment using PF06650833, the IRAK4i now being tested in clinical trials for patients with rheumatoid arthritis (35) (NCT02996500, ClinicalTrials.gov). In this experiment, CRC-bearing mice were sacrificed when their subcutaneous xenograft tumors exceeded $2000 \mathrm{~mm}^{3}$, or when any humane endpoints predefined in the animal protocol were met. In both 
A $\quad$ DMSO oxali. $10 \mu \mathrm{M}$

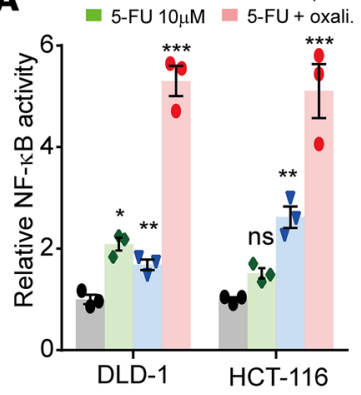

B

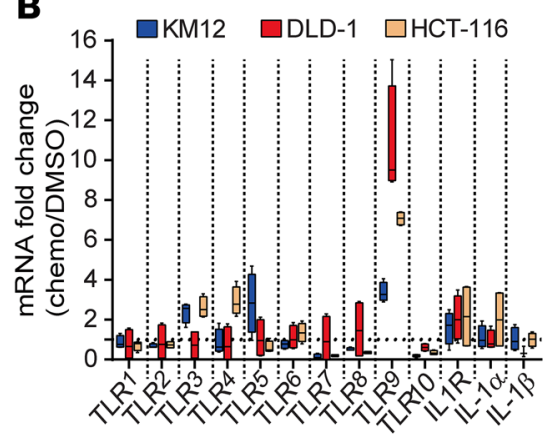

C

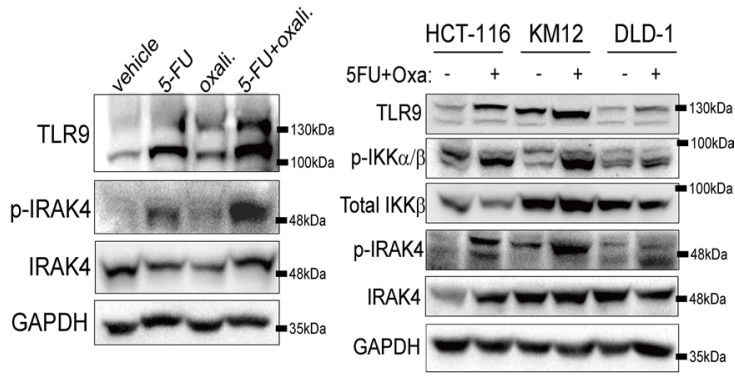

PLA: TLR9+p-IRAK4/DAPI
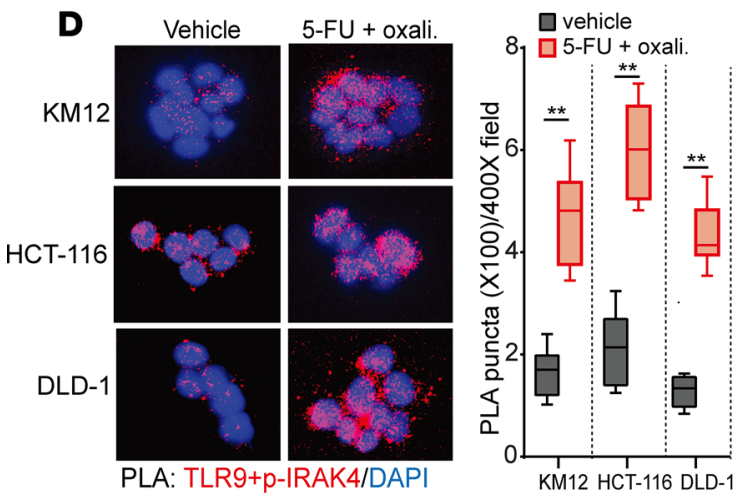

$\mathbf{E}$

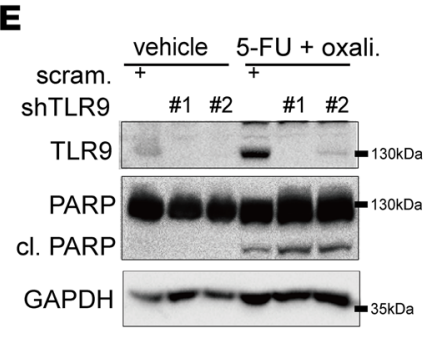

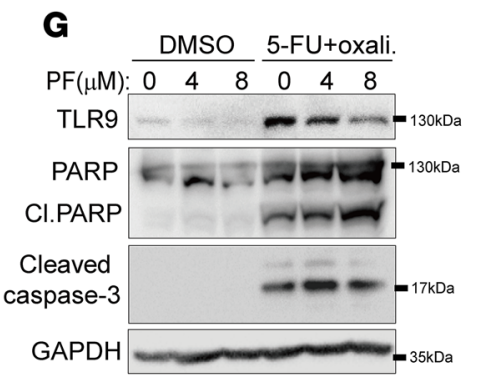
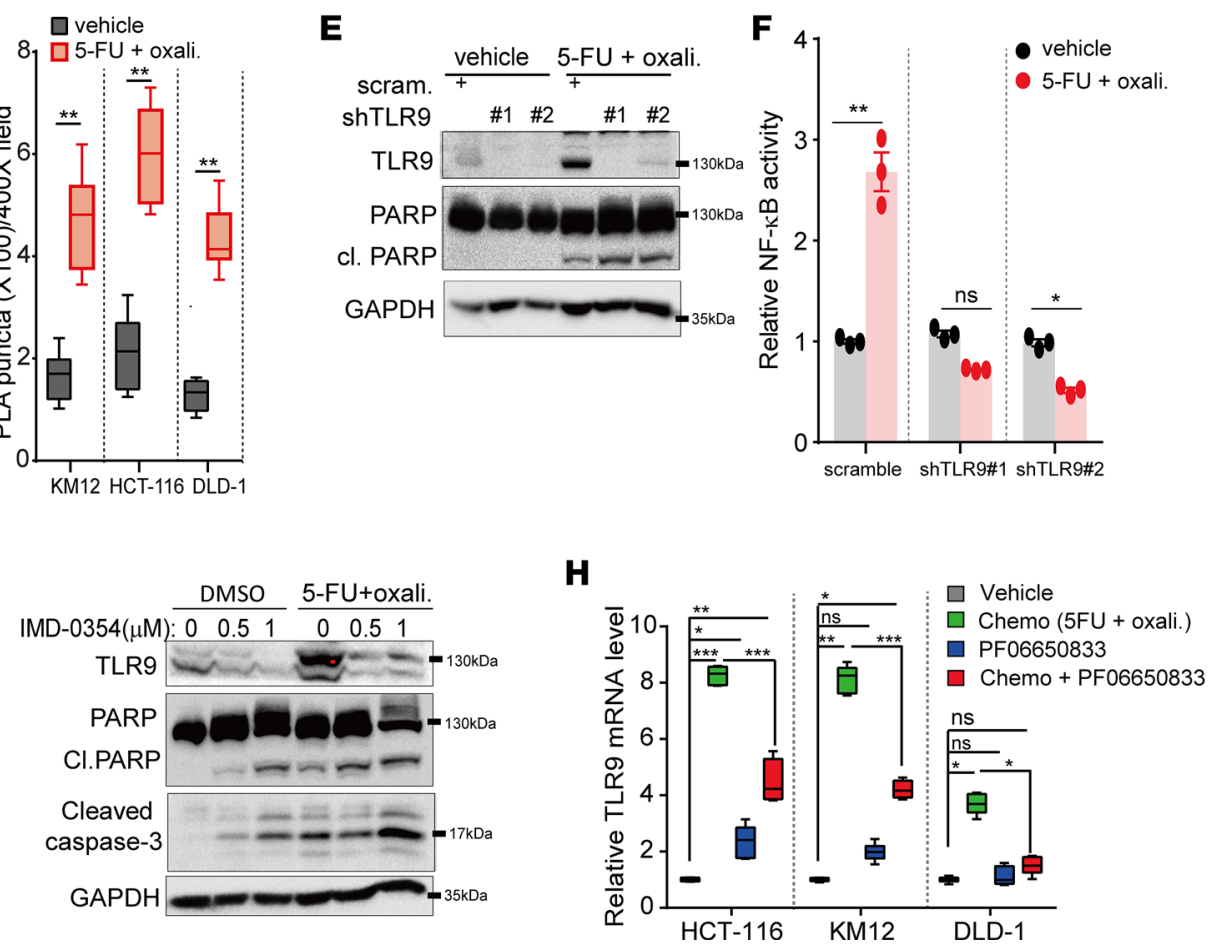

Figure 6. Chemotherapy induces autologous TLR9-IRAK4-NF-KB circuitry. (A) Luciferase reporter assay showing the effect of chemotherapy on NF- $\kappa B$ activity in 2 different CRC lines. Data represent 1 of 3 sets of experiments each done in triplicate and presented as mean \pm SEM (ANOVA, ${ }^{*} P<0.05,{ }^{* *} P<0.01,{ }^{* * *} P$ $<0.001$ ). (B) Quantitative PCR showing changes in expression of the indicated genes in 3 CRC lines following exposure to chemotherapy (5-FU and oxaliplatin, both $10 \mu \mathrm{M}$ ) overnight. Data represent 1 of 2 sets of experiments each done in biological duplicates and technical triplicates and presented as mean \pm SEM. (C) Western blots confirming increased protein expression of TLR9 following overnight treatment with the indicated chemotherapeutic agent (all at $10 \mu \mathrm{M}$ ) in HCT-116 (left panel) and 3 different CRC lines (right panel). (D) Representative $(\times 400)$ confocal images and quantification (mean \pm SEM) of proximal ligation assay (PLA) puncta (red) formed between TLR9 (CST \#D9M9H) and p-IRAK4 (ABNOVA \#A8A8, 1:500) in 3 colon lines treated with vehicle or chemotherapy (5-FU and oxaliplatin, both $10 \mu \mathrm{M}$ ) overnight (2-tailed $t$ test, ${ }^{* *} P<0.01$ ). (E) Western blots showing the effect of TLR9 knockdown on chemotherapy-induced PARP cleavage in HCT-116 cells. (F) Luciferase reporter assay showing the effect of TLR9 knockdown on chemotherapy-induced NF- $\mathrm{KB}$ activity in HCT-116 cells (ANOVA, ${ }^{*} P<0.05,{ }^{* *} P<0.01$ ). (C) Western blots showing suppressive effects of IRAK4 and IKK inhibitors on chemotherapy-induced TLR9 expression and apoptosis. (H) Quantitative PCR showing suppressive effect of IRAK4i (PF06650833, $4 \mu$ M overnight) on chemotherapy-induced TLR9 expression. Data represent 1 of 2 sets of experiments each done in biological duplicates and technical triplicates and presented as mean \pm SEM (Tukey's multiple-comparisons test, $\left.{ }^{*} P<0.05,{ }^{* *} P<0.01,{ }^{* *} P<0.001\right)$.

models, we observed significantly slower tumor growth kinetics and prolongation of survival in mice treated with PF06650833 plus chemotherapy (Figure 7, F and J). Combination-treated mice had slower weight gain compared with mice in other arms over the treatment course but did not exhibit any noticeable signs of distress (Supplemental Figure 6, B and C). Together, our results strongly support testing IRAK4i with chemotherapy in clinical trials for CRC patients.

High $p$-IRAK4 is associated with poor overall survival in colon cancer patients. For colon cancer patients with stage III (lymph node-positive) and stage II disease with high-risk histopathologic features (particularly T4 disease, or stage IIb, IIc), 5-FU plus oxaliplatin (FOLFOX) is routinely administered after surgery to 
A

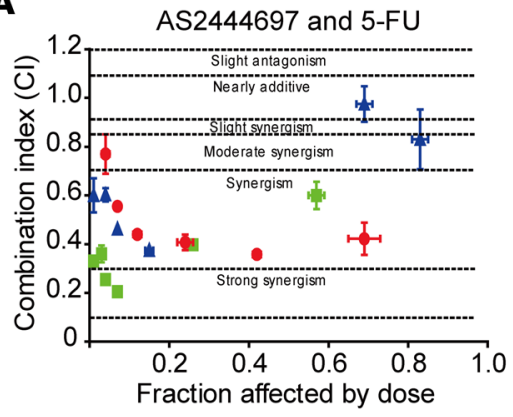

B

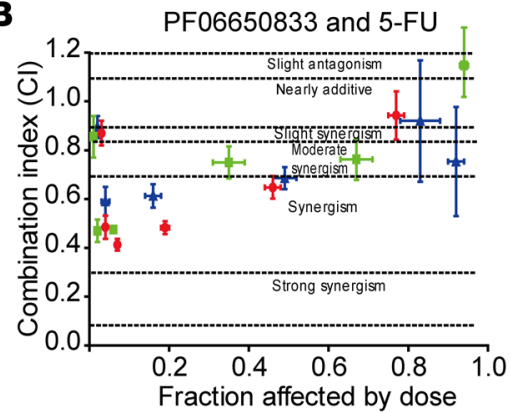

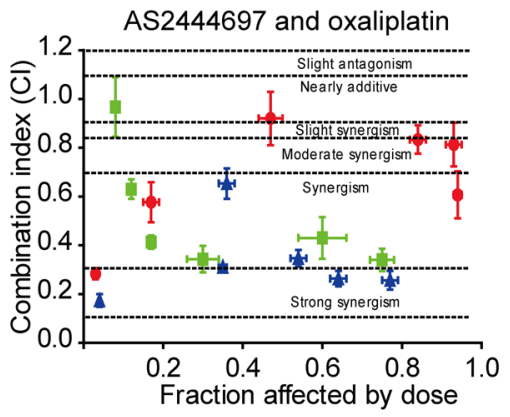

PF06650833 and oxaliplatin

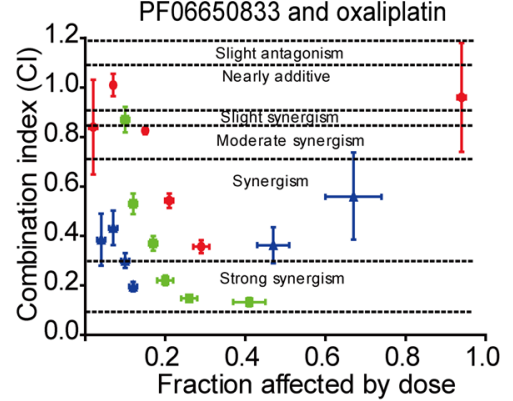

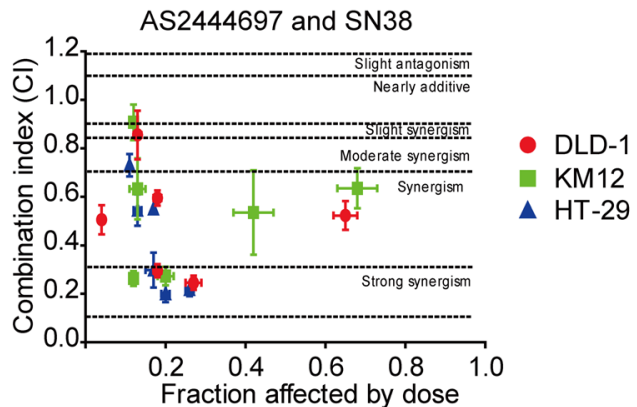

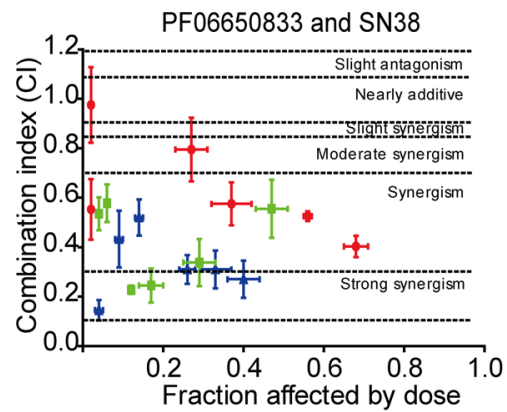

\section{DLD-1 Model}
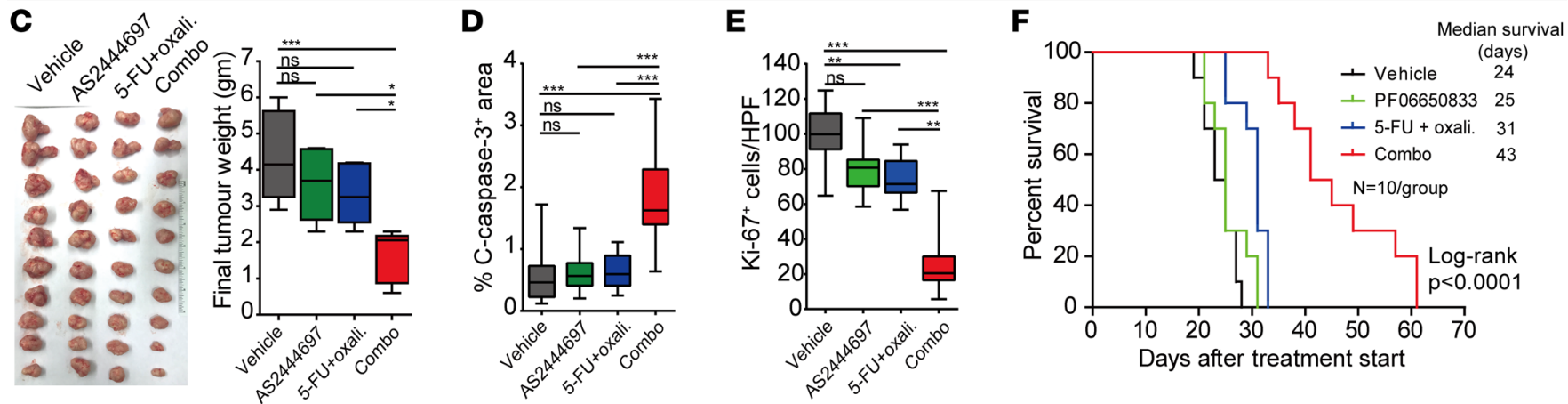

KM12 Model
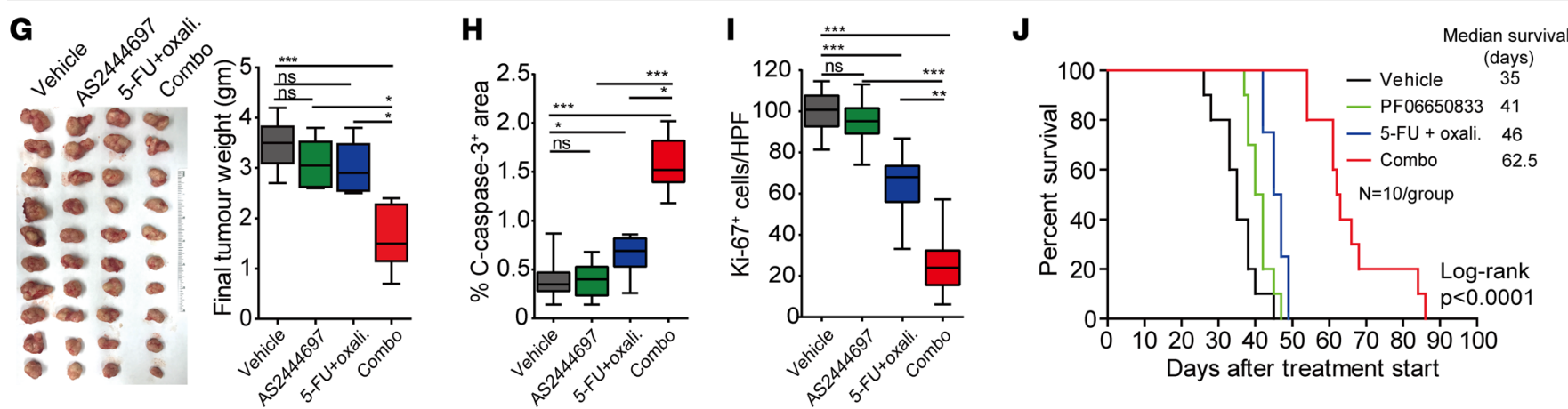

Figure 7. IRAK4 inhibition potentiates the cytotoxic effect of chemotherapy in vivo. (A and B) Combination indices between AS2444697 (A) or PF06650833 (B) and chemotherapeutic agents 5-FU, oxaliplatin, and SN-38 in 3 different CRC lines calculated using CompuSyn software. Cells were cultured in 6 fixed-ratio concentrations in triplicate over 72 hours and viability assayed by Alamar Blue. The concentrations (in $\mu M$ ) of IRAK4 inhibitor and each chemotherapeutic agent (5-FU or oxaliplatin or SN-38) were 8 and 50, 4 and 25,2 and 12.5, 1 and $6.25,0.5$ and 3.125, and 0.25 and 1.56. Experiments were done 3 times in triplicate, and 1 set of data is presented as mean \pm SEM. (C) Final weight and picture of DLD-1 tumors harvested simultaneously when vehicle-treated mice reached maximum volume of approximately $2000 \mathrm{~mm}^{3}$. Data represent mean \pm SEM (Tukey's multiple-comparisons test, ${ }^{*} P<0.05,{ }^{* * *} P<0.001$ ). (D and E) Quantification of cleaved caspase- $3^{+}$area per $\times 200$ field $(\mathbf{D})$ and dual $\mathrm{CK}^{+}$and $\mathrm{Ki}^{-67^{+}}$ cells per $\times 400$ field (E) of DLD-1 tumors treated as indicated. Ten random pictures were taken from each 10 tumors per arm, and data represent mean \pm SEM (Tukey's multiple-comparisons test, ${ }^{* *} P<0.01,{ }^{* *} P<0.001$ ). (F) Kaplan-Meier survival of mice bearing DLD-1 tumors treated as indicated when tumors reached $100 \mathrm{~mm}^{3}$ and until tumor volume exceeded $2000 \mathrm{~mm}^{3}$ or whenever humane endpoints were reached $(n=10$ per group, log-rank test). (G) Final weight and picture of KM12 tumors harvested simultaneously when vehicle-treated mice reached maximum volume 
of approximately $2000 \mathrm{~mm}^{3}$. Data represent mean \pm SEM (Tukey's multiple-comparisons test, ${ }^{*} P<0.05,{ }^{* * *} P<0.001$ ). (H and $\left.\mathbf{I}\right)$ Quantification of cleaved caspase $-3^{+}$area per $\times 200$ field $(\mathbf{H})$ and dual $\mathrm{CK}^{+}$and $\mathrm{Ki}-67^{+}$cells per $\times 400$ field (I) of KM12 tumors treated as indicated. Ten random pictures were taken from each 10 tumors per arm, and data represent mean \pm SEM (Tukey's multiple-comparisons test, ${ }^{*} P<0.05$, ${ }^{* *} P<0.01,{ }^{* * *} P<0.001$ ). (J) Kaplan-Meier survival of mice bearing KM12 tumors treated as indicated when tumors reached $100 \mathrm{~mm}^{3}$ and until tumor volume exceeded 2000 $\mathrm{mm}^{3}$ or whenever humane endpoints were reached ( $n=10$ per group, log-rank test).

lower the chance for recurrence. However, up to $30 \%$ of treated patients still develop relapse, indicating resistance of micro-metastatic cells to chemotherapy. Since our preclinical studies showed that activated IRAK4 may be a mechanism of chemoresistance, we reasoned that high IRAK4 activity may be associated with poorer prognosis in patients with stage IIb-III disease who are routinely treated with FOLFOX. To this end, we analyzed p-IRAK4 IHC staining on 2 commercial CRC TMAs with annotated overall survival data. In these CRC samples, p-IRAK4 staining was present predominantly in the cytoplasm of neoplastic cells, while some staining was seen in infiltrative leukocytes and a subset of stromal cells (Figure 8A and ref. 42). We found that high p-IRAK4 staining in neoplastic cells was significantly associated with poorer overall survival in patients with stage IIb-III disease, but not in patients with stage I or II disease (Figure 8, B-D). Notably, analysis of The Cancer Genome Atlas (TCGA) database showed that the overall survival of stage IIb-III colon cancer patients with tumors expressing higher IRAK4 mRNA levels, as defined by mRNA $Z$ score greater than 0.5 , was significantly poorer than that of the remaining patients with lower IRAK4 mRNA expression (Figure 8E). In patients with stage IV CRC, FOLFOX in combination with an anti-VEGF or anti-EGFR antibody is frequently administered as first-line treatment, but the response rate is about $50 \%-60 \%$. To probe the prognostic impact of p-IRAK4 in metastatic CRC patients, we collected metastatic liver samples from 225 patients who underwent synchronous or metachronous liver and colon resection with curative intent at our institution. TMAs were constructed from the metastatic liver tumors from these patients and stained with p-IRAK4 antibody. All samples were categorized as high, medium, or low in p-IRAK4 staining by $\mathrm{H}$ score. Thirty-two patients had paired primary and metastatic liver CRC tumors for comparison. We did not observe significant correlation in p-IRAK4 staining intensity between paired primary and metastatic liver samples (Figure $8 \mathrm{~F}$ ). The remaining 204 patients went on to receive systemic chemotherapy at either Washington University or other institutions. Notably, when controlling for other clinicopathologic factors (Supplemental Table 1), we found that patients with high tumor p-IRAK4 IHC staining had significantly poorer overall survival compared with those with medium to low p-IRAK4 levels (Figure 8G). Overall, these data show that high IRAK4 activity is a poor prognostic factor for CRC patients, lending further support for targeting IRAK4 in combination with chemotherapy in CRC to improve outcome.

\section{Discussion}

In the present study, we provide evidence, for the first time to our knowledge, that IRAK4 is activated and drives IKK-NF- $\mathrm{kB}$ activity and is a novel promising therapeutic target in CRC. Using pharmacologic and genetic approaches, we showed that targeting IRAK4 stifles colitis-induced neoplastic progression in a genetic mouse model and lowers NF- $\mathrm{BB}$ activity in human CRC cells. We provide new mechanistic insight that chemotherapy induces a TLR9-IRAK4-NF- $\mathrm{B}$ feedback loop in CRC cells that, when disrupted, can enhance chemotherapy-induced apoptosis. On this premise, IRAK4 inhibitors synergize with chemotherapy and prolong survival of CRC-bearing mice. Notably, activation or high expression of IRAK4 is predictive of poorer overall survival in colon cancer patients who receive chemotherapy. As opposed to IKK or TAK1, targeting of IRAK4 may be more clinically tractable, based on lack of observed toxicities in our treated mice and that IRAK4-null mice are viable and have normal lifespan (43). However, both IRAK4 inhibitors that we used had no single-agent activity in CRC growth in vivo, indicating the need to combine with chemotherapy in future clinical trial design for CRC patients. In addition, all combination-treated mice eventually still succumbed to disease progression, suggesting emergence of resistance mechanisms that remain to be investigated. Importantly, because IRAK4-null mice are defective in mounting innate immunity when challenged with microbes (43), and IRAK4-deficient patients suffer from severe bacterial infections before puberty (44, $45)$, infection will be a concern for IRAK4 inhibitors especially when coadministered with chemotherapy. Therefore, proper antimicrobial prophylaxis should be considered in future clinical trial design.

In this study, we showed TLR9 to be the driver of IRAK4 and NF- $\mathrm{BB}$ in CRC cells upon chemotherapy exposure. Interestingly, we also observed significantly upregulated TLR9 mRNA expression in pancreatic cancer cells (MIA Paca-2) following exposure to oxaliplatin, SN-38, 5-FU, and gemcitabine, suggesting that 
A

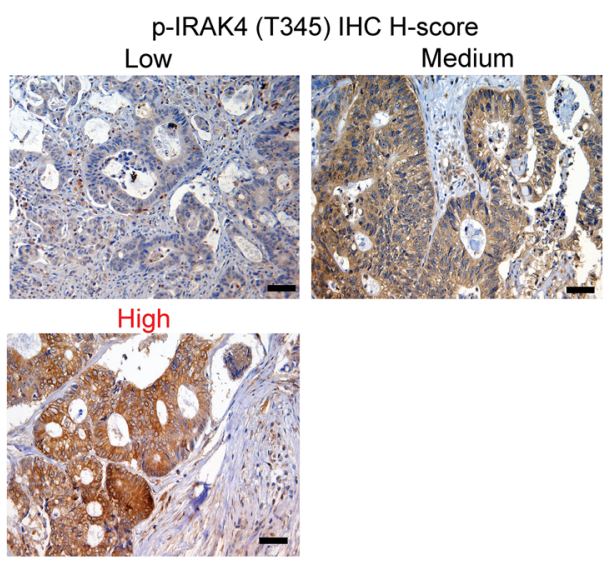

B

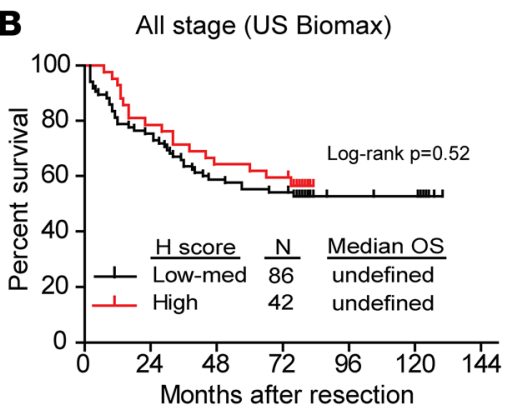

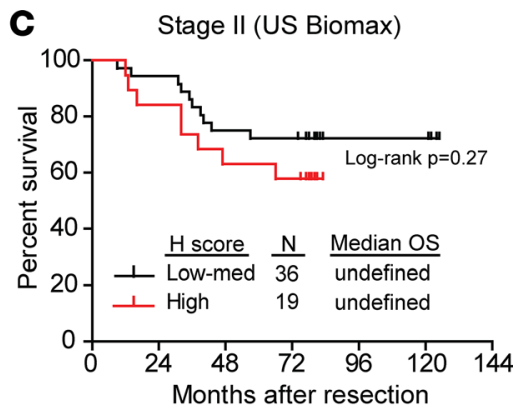

D

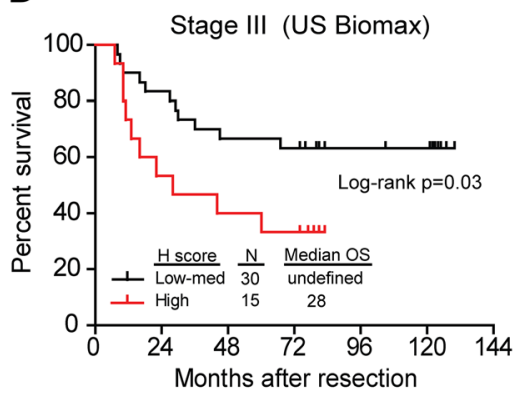

E

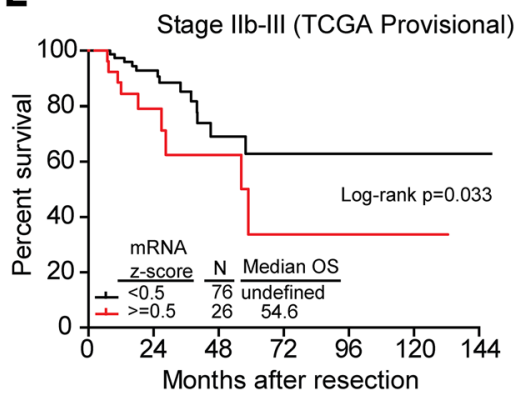

$\mathbf{F}$

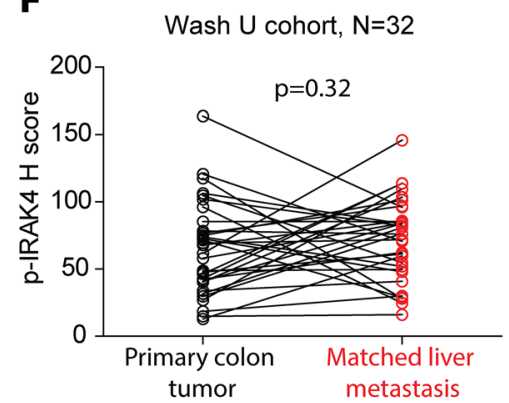

G

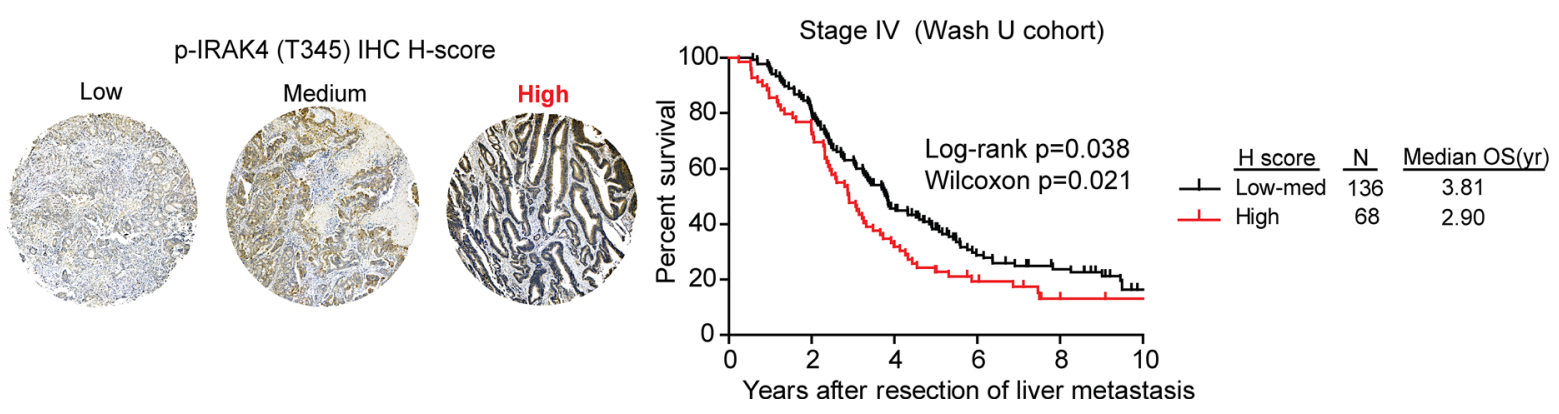

Figure 8. Prognostic impact of IRAK activation or expression in colon cancer. (A) Representative IHC images showing different intensities of p-IRAK4 staining as determined by $\mathrm{H}$ score from 2 commercial colon cancer TMAs (US Biomax BC051110b, HCol-Ade180Sur-08) (scale bars: $100 \mu \mathrm{m}$ ). (B-D) The overall survival (OS) by Kaplan-Meier analysis of patients with different stages of colon cancer as stratified by the $\mathrm{H}$ score of $\mathrm{p}$-IRAK4 staining of 2 commercial colon cancer TMAs (US Biomax BC051110b, HCol-Ade180Sur-08). (E) Overall survival by Kaplan-Meier analysis of stage IIb-III colon cancer patients as stratified by the IRAK4 mRNA expression ( $Z$ score cutoff at 0.5 ) from TCGA (provisional) database. (F) Correlation (Wilcoxon signed-rank test) of p-IRAK4 IHC staining intensity between matched primary and liver metastatic CRC samples from a cohort of 32 patients who underwent surgery at Washington University from years 2000 to 2010 . (G) Representative IHC images showing different intensities of p-IRAK4 IHC staining in liver metastasis samples, and Kaplan-Meier overall survival, as stratified by p-IRAK4 H score from a cohort of 204 patients who underwent liver resection surgery at Washington University from years 2000 to 2010.

TLR9 upregulation may be a common adaptive response following DNA damage at least in these 2 cancer types (Supplemental Figure 7). Based on these premises, we propose that acute DNA damage response recruits ATM, which directly triggers the NF- $\mathrm{kB}$ cascade, while damaged DNA may also activate the preexisting pool of TLR9. Activation of the NF-KB cascade results in transcriptional upregulation of TLR9, which subsequently engages the IRAK4-NF- $\mathrm{KB}$ cascade to amplify and sustain the feed-forward circuitry (Figure 9). Besides TLR9, it is reasonable to hypothesize that 1 or more TLRs, and their natural ligands originating from surrounding damaged cells or even gut microbes within the tumor microenvironment, are the trigger of IRAK4 and NF- $\mathrm{KB}$ in CRC. Supporting this notion, manipulation of gut microbiome composition with fecal transfer or antibiotics can enhance or lower colitis-induced CRC formation in mice $(46,47)$, establishing microbiota dysbiosis as a protumorigenic event. More provocatively, transfer of fecal 


\section{Model: DNA damage induces TLR9-IRAK4-NF-kB survival mechanism}
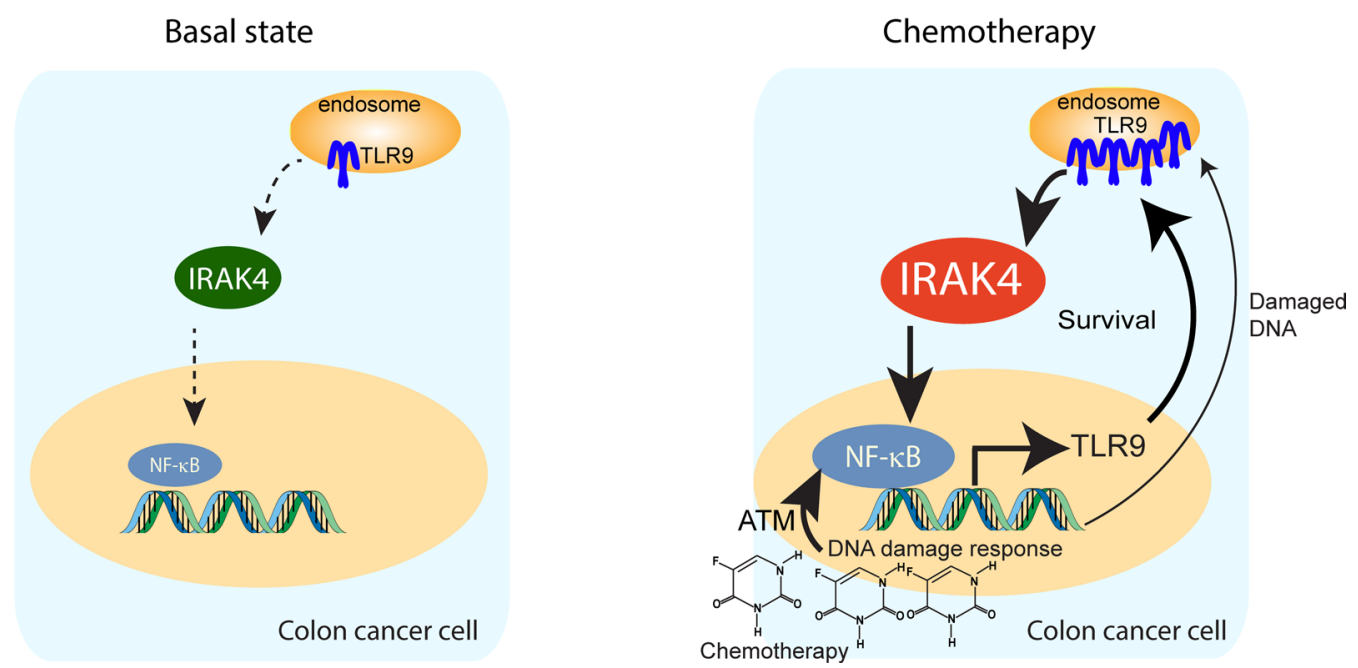

Figure 9. Schematics depicting a proposed model in which DNA damage incurred by chemotherapy triggers a TLR9IRAK4-NF- $\kappa B$ circuitry to sustain CRC cell survival. At basal state, IRAK4 and NF- $\kappa B$ are activated through mechanisms that remain to be determined, but likely through upstream TIR family members including TLR9. Upon exposure to chemotherapy, damaged DNA may activate NF- $\mathrm{KB}$ through ATM and engage the preexisting pool of TLR9, which in turn activates IRAK4 and NF-KB, resulting in survival and further upregulation of TLR9. This chain of events results in enhanced survival, which enables CRC cells to evade chemotherapy-induced apoptosis.

samples from CRC patients promotes intestinal inflammation and tumorigenesis in germ-free mice (48). Because IRAK4 is the master kinase downstream of most TLRs, it will be very interesting to determine whether IRAK4 inhibition can suppress CRC formation in these models.

The actual role of MyD88, the critical adaptor that recruits and activates IRAK4, during different stages of colitis-induced CRC development is divergent, largely dependent on mouse models and experimental conditions (49). We showed that IRAK4i is highly effective in blocking DSS-induced neoplastic progression in $A P C^{\mathrm{Min} /+}$ mice but has no effect on tumorigenic growth of established CRC tumors in vivo. To clearly determine the role of epithelial IRAK4 during neoplastic progression, we pretreated $A P C^{\mathrm{Min} /+}$ mice with DSS to allow enough time for inflammation to occur before introduction of IRAK4i, which is known to have antiinflammatory effect. The near-complete obliteration of tumor formation in IRAK4i-treated mice suggests epithelial IRAK4 to be required in neoplastic transformation, which was further supported by the requirement of IRAK4 in the MC38 model. These results are in agreement with work by others that showed an essential cell-intrinsic role of MyD88 in spontaneous intestinal and azoxymethane-induced (AOM-induced) colon cancer formation in $A P C^{\mathrm{Min} /+}$ mice (50-52). As opposed to the $M y D 88^{-1-} A P C^{\text {Min } /+}$ model, $M y D 88^{-1-} \mathrm{C} 57 \mathrm{BL} / 6$ mice develop more severe colitis upon acute AOM and/or DSS treatment, leading to more DNA damage and higher incidence of CRC development $(50,53,54)$. On the contrary, in a chronic oxazolone-induced colitis model, $M y D 88^{-1-}$ mice develop robust inflammation but not colon cancer, largely because of loss of MyD88-dependent protumorigenic M2 macrophages (55). Nonetheless, coadministration of MyD88 inhibitor (TJ-M2010-5) with DSS after AOM treatment attenuated colitis and prevented CRC development in BAL$\mathrm{B} / \mathrm{c}$ mice (56). Importantly, we showed that IRAK4 function in hematopoietic cells is critical in DSS-induced colitis and CRC progression, although no difference in leukocyte abundance was seen throughout the colon

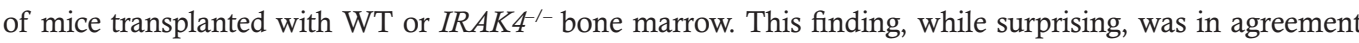
with another report that showed that $L y s M$-Cre-driven deletion of IKK $\beta$ in myeloid cells reduces colitis-induced tumor burden without affecting the abundance of myeloid infiltrates (10). We envision the following possibilities: First, loss of IRAK4 may attenuate DSS-induced and/or subsequent tumor-associated inflammation, allowing faster healing of injured epithelial cells. Second, loss of hematopoietic IRAK4 reduces/ abrogates production of tumor-promoting cytokines such as IL-1 $\beta$ and TNF- $\alpha$, which have been shown to be regulated by $\operatorname{IKK} \beta(10)$, which may directly impact epithelial cell transformation and proliferation. Third, loss of hematopoietic IRAK4 may shift the composition of intratumoral immune infiltrates, including subtypes of macrophage, granulocyte, and lymphocyte subsets, to an antitumorigenic phenotype, thereby impeding 
neoplastic progression. Generation of conditional IRAK4-null mice will be essential in dissecting the role of IRAK4 in various subsets of hematopoietic cells during CRC development and to definitively address these possibilities. Nonetheless, our results suggest that systemic inhibition of IRAK4 appears to be an effective strategy in curbing CRC development.

Lastly, we showed that activated IRAK4 (p-IRAK4) is associated with poor prognosis in CRC patients with stage IIb-IV disease after resection. These patients are routinely treated with chemotherapy (mainly FOLFOX) following surgery, and therefore high p-IRAK4 is a potential indicator for lack of benefit from chemotherapy. Our results are congruent with another report that showed that overexpression of MyD88 is associated with poor prognosis in colon cancer (57). Importantly, it is well appreciated that only about $20 \%$ of patients with stage III CRC benefit from adjuvant FOLFOX (58). While various combinations of markers have been developed to aid prognostication and patient selection for chemotherapy (59), enhancing the effect of chemotherapy, as can be achieved by cotargeting of IRAK4 and IKK-NF- $\mathrm{BB}$, represents an equally important endeavor to improve overall patient outcome.

In summary, our study provides preclinical rationale for testing IRAK4 inhibitors in combination with chemotherapy in CRC. We demonstrate that chemotherapy can induce IKK-NF- $\mathrm{BB}$ and sustain CRC cell survival by upregulating TLR9/IRAK4 signaling, providing justification for targeting IRAK4. Further work is needed to elucidate potential resistance mechanisms and to enable development of more effective and durable combinatorial regimens for this promising treatment paradigm.

\section{Methods}

Cell lines. All human cell lines were purchased from ATCC, which performs its own authentication by short tandem repeat DNA profiling. The MC38 cell line was a gift from David DeNardo (Washington University in St. Louis [WUSTL], St. Louis, Missouri, USA). SW620, SW620-L1, and SW620-L2 were gifts from Mien-Chie Hung (MD Anderson Cancer Center, Houston, Texas, USA) and were used as previously described (60) and were not further authenticated. All cells were cultured in DMEM/10\% FBS/1\% penicillin-streptomycin at $5 \% \mathrm{CO}_{2}, 37^{\circ} \mathrm{C}$ incubators. Mycoplasma testing was performed every 6 months using MycoSEQ Detection kit (Applied Biosystems). All cell lines were used for less than 6 months after receipt or resuscitation from cryopreservation.

Human and murine CRC samples and tissue microarrays. All studies were performed according to ethical principles of the Declaration of Helsinki. Commercial CRC tissue microarray (TMA) sections were purchased from US Biomax (CO702a, C0952a, BC05002b, BC051110b, HCol-Ade180Sur-08). All cases were deidentified. Human colorectal TMA was established at Washington University from 225 patients with metastatic CRC who underwent synchronous or metachronous resection with curative intent from years 2000 to 2010. The TMA contained 2 cores (1.0 mm diameter) from each patient. Clinical information of these patients has been maintained in a retrospective clinical database. For survival analyses of Washington University CRC TMA, 21 patients were excluded as a result of poor sample quality ( $>50 \%$ area shredded or necrotic, $n=8$ ), demise within 3 months after surgery from postsurgical complications $(n=5)$, or having no documentation on subsequent chemotherapy $(n=8)$. The remaining 204 patients went on to receive systemic chemotherapy at either Washington University or other institutions.

Mouse experiments. For xenograft experiments, 5 million cells per flank were injected s.c. into 7 to 8-week-old nude mice (for human cells) or C57BL/6J mice (for MC38 cells). Both mouse strains were purchased from The Jackson Laboratory. Treatment was started when tumors reached a volume of approximately $100 \mathrm{~mm}^{3}$. APC $C^{\mathrm{Min} /+}$ mice were a gift from Nicholas Davidson, WUSTL, originally from The Jackson Laboratory. IRAK4-null mice were purchased from EUCOMM. Both strains were maintained and genotyped per vendor's protocol. For the colitis-induced CRC model, 6- to 8-week-old $A P C^{\mathrm{Min} /+}$ mice (gift from Nicholas Davidson, WUSTL, originally from The Jackson Laboratory) were treated for 1 week with $2 \%$ DSS in drinking water, which was then replaced with regular drinking water. All mice were euthanized 4 weeks later, and colons were prepared and analyzed as published (61). Histologic analyses of mouse colons were independently performed by KHL, QL, and gastrointestinal pathologist MBR in a blinded manner. For bone marrow transplantation, 6-week-old $A P C^{\mathrm{Min} /+}$ recipient mice were given a split dose ( $\sim 4$ hours apart) of lethal irradiation totaling 10.5 Gy prior to transplantation via retro-orbital injection of $2 \times 10^{6}$ unfractionated whole bone marrow cells harvested from 8-week-old WT and IRAK4-null mice. 
Immunohistochemistry, immunofluorescence, and scoring. Antibodies used for IHC and immunofluorescence (IF) staining are provided in Supplemental Table 2. Unless otherwise specified, all IF quantification was performed under fluorescence or light microscopy on ten $\times 20$-power fields per tumor, and data are presented as mean \pm SEM, as previously described (26). For all TMA image analysis, whole slide tissue scans were obtained at $\times 20$ magnification on a Zeiss Axio Scan Z1 Brightfield/Fluorescence Slide Scanner (resolution $0.645 \mu \mathrm{m}$ per pixel). Whole tissue slide scans were then analyzed with HALO software (Indica Labs Perkin Elmer) using TMA module with Area quantification FL V1.2. P-IRAK4 IHC intensity for each sample was calculated using $\mathrm{H}$ score $[1 \times(\%$ of lightly stained cells $)+2 \times(\%$ of intermediate stained cells $)+3$ $\times\left(\%\right.$ of darkly stained cells)]. Tissue cores with exhausted epithelium were excluded. For $A P C^{\text {Min } /+}$ mouse experiments, histologic analyses and colitis scoring were conducted using published methods (62). Briefly, a 16-point system consisting of crypt damage (scale, 0-4), crypt damage extent (scale, 0-4), inflammatory infiltrate (scale, 0-4), and inflammatory infiltrate extent (scale, 0-4) was applied to 3 random $\times 400$ fields in mid- and distal colons of each mouse. Staining intensity obtained from scanners was independently verified by gastrointestinal pathologist MBR. Survival analysis was performed by JL in a blinded manner.

Plasmids and creation of stable cell lines. Stable knockdowns were generated using pSuperRetro as previously described (26). Target sequences were: shIRAK4\#1, TTCAGTAGTAATGTCAACC; shIRAK4\#2, GCCTAATGGTTCATTGCTA; shTLR9\#1, GAGCTAAACCTGAGCTACAAC; shTLR9\#2, GCACGGTGCCACCTCCACACT. Mouse CRISPR sequences were: sgIRAK4\#1, GTCGCCAACTGTGCAGTTCG; sgIRAK4\#2, TGGCGACCTTGTGGATCTAC.

Drugs and reagents. SN-38, 5-FU, and oxaliplatin were purchased from the Siteman Cancer Center Pharmacy. The IRAK4 inhibitors PF06650833 (catalog 6373) and AS2444697 (catalog 5430) and the IKK $\beta$ inhibitor IMD-0354 (catalog 2611) were purchased from Tocris.

$N F-\kappa B$ reporter assays. NF- $\kappa$ B reporter assays were done using Dual-Glo Luciferase Assay System and read with Synergy H4 Hybrid Microplate Reader.

Western blots. Cell lysates were harvested using radioimmunoprecipitation assay (RIPA) cell lysis buffer (10 mM Tris- $\mathrm{HCl}$ [pH 7.4], $50 \mathrm{mM} \mathrm{NaCl}, 0.05 \%$ SDS, $10 \mathrm{mM} \mathrm{Na}$-EDTA, 0.5\% Triton X-100, 0.5\% sodium deoxycholate, supplemented with protease and phosphatase inhibitors [Roche]). Cell lysates were resolved in Tris-glycine SDS-PAGE gels and transferred to PVDF membranes (Invitrogen). Membranes were probed with primary antibodies diluted in blocking buffer $(1 \times$ TBST with $5 \% \mathrm{wt} / \mathrm{vol} \mathrm{BSA})$ overnight at $4^{\circ} \mathrm{C}$. The primary antibodies are listed in Supplemental Table 2. Membranes were washed 3 times in $1 \times$ TBST buffer and probed with HRP-conjugated secondary antibody for 1 hour at room temperature. Membranes were developed with the Pierce ECL Western Blotting Substrate and detected using a ChemiDoc XRS+ system (Bio-Rad). Full, uncut gels are provided in the published online supplemental material.

In vitro cell viability assay and calculation of combination indices. Five thousand cells per well were plated in triplicate in 96-well plates 1 day before addition of the inhibitors at the indicated final concentrations. After 5 or 7 days of culture, viability assay was measured using resazurin colorimetric analysis as previously described (26). For drug interaction studies, cells were cultured in triplicate in the presence of 6 fixed-ratio concentrations for 72 hours followed by Alamar Blue viability assay. Combination indices were calculated using CompuSyn software as previously described (41). All experiments were done at least 3 times in triplicate, and 1 most representative set of data was presented.

Proximal ligation assay. Proximal ligation assay was performed using Duolink in situ starter kit (DUO92101, Sigma-Aldrich) per manufacturer's protocol. Number of puncta per field was quantitated with NIS-Elements software using a Nikon C2+ fluorescent confocal microscope. Ten random $\times 400$ fields per condition were analyzed and quantitated.

Public database. For TCGA data, matching clinical and IRAK4 mRNA expression data on Colorectal Adenocarcinoma (TCGA, provisional, 640 samples) were downloaded from the cBioPortal website (63). For IRAK4 expression, RNA-Seq V2 RSEM data set was used. Patients with missing survival data, clinical stage, or IRAK4 expression data or who were known not to have received adjuvant treatment or died less than 3 months from initial diagnosis were excluded. Clinical stage was determined based on provided information, or the provided tumor, nodal, and metastasis information. Rectal cancer was excluded, since these patients typically received neoadjuvant chemoradiation, which poses an additional variable. Kaplan-Meier survival analysis was independently performed by biostatistician $\mathrm{JL}$, after separation of patients based on IRAK4 mRNA expression level ( $Z$ score cutoff 0.5 , which roughly separates all cases to upper 1 and lower 3 quantiles). Expression profiles of IRAK4 in normal colon versus cancer were downloaded from the Oncomine database (33). 
Statistics. All results, when applicable, were expressed as the means \pm SEM. Statistical analysis was performed using the Prism 6 software program. Unpaired Student's 2-tailed $t$ tests were used to compare 2 groups when appropriate. For multiple groups, 1-way ANOVA with Tukey's post-test was used. $P$ values less than 0.05 were considered as statistically significant. Cox proportional hazards models were used to evaluate the relationships between clinical characteristics and overall survival. The upper quantile of p-IRAK4 H score in TMA and IRAK4 mRNA in TCGA was used as cut point. Survival probabilities were calculated using Kaplan-Meier method. Differences between strata of p-IRAK4 H score and IRAK4 mRNA were determined by log-rank tests. All statistical tests were 2-sided using an $\alpha=0.05$ level of significance. SAS version 9.4 was used to perform all survival analyses.

Study approval. All mouse experiments were conducted under approval by WUSTL IACUC (protocol 20160142). We adhered to the ARRIVE guidelines for reporting on animal studies. All human tissue studies were approved by the Washington University School of Medicine Ethics Committee (IRB 201108117).

\section{Author contributions}

QL and KHL designed the studies and wrote the manuscript. QL, YC, DZ, LL, PMG, NK, and HJ conducted the experiments and acquired and analyzed data. GAC performed bone marrow transplant experiments. JG and RCF constructed the CRC TMA and provided clinical data on patients. JH and DGD performed automated TMA data acquisition and computation of $\mathrm{H}$ scores of all TMAs. MBR independently analyzed all TMAs and tissue sections in a blinded manner. JL performed survival analysis on TCGA and TMA data sets in a blinded manner. KHL supervised the entire project.

\section{Acknowledgments}

This study was supported by NIH grant R21 CA223112 (to KHL and MBR), the BJHF-ICTS Clinical and Translational Research Program (to KHL), Concern Foundation Conquer Cancer Award 388329 (to KHL), the SIP Award funded by the Siteman Cancer Center and the Foundation for BJH (to KHL and MBR), Surgical Oncology NCI T32 training grant (to JG), and NIH CTSA Grant UL1 TR000448 (to KHL). QL is supported by the National Natural Science Foundation of China (grant 81401735). We acknowledge the WUSTL Digestive Diseases Research Cores Center (grant P30 DK052574) for providing technical support.

Address correspondence to: Kian-Huat Lim, Washington University School of Medicine, 660 South Euclid Avenue, Campus Box 8069, Saint Louis, Missouri 63110, USA. Phone: 314.362.6157; Email: kian-huat.lim@wustl.edu.

1. Siegel RL, Miller KD, Jemal A. Cancer statistics, 2019. CA Cancer J Clin. 2019;69(1):7-34.

2. Bray F, Ferlay J, Soerjomataram I, Siegel RL, Torre LA, Jemal A. Global cancer statistics 2018: GLOBOCAN estimates of incidence and mortality worldwide for 36 cancers in 185 countries. CA Cancer J Clin. 2018;68(6):394-424.

3. Hammond WA, Swaika A, Mody K. Pharmacologic resistance in colorectal cancer: a review. Ther Adv Med Oncol. 2016;8(1):57-84.

4. Wang CY, Cusack JC, Liu R, Baldwin AS. Control of inducible chemoresistance: enhanced anti-tumor therapy through increased apoptosis by inhibition of NF-kappaB. Nat Med. 1999;5(4):412-417.

5. Wu D, et al. NF- $\mathrm{kB}$ expression and outcomes in solid tumors: a systematic review and meta-analysis. Medicine (Baltimore). 2015;94(40):e1687.

6. Sakamoto K, et al. Constitutive NF- $\mathrm{kB}$ activation in colorectal carcinoma plays a key role in angiogenesis, promoting tumor growth. Clin Cancer Res. 2009;15(7):2248-2258.

7. Lind DS, et al. Nuclear factor-kappa B is upregulated in colorectal cancer. Surgery. 2001;130(2):363-369.

8. Kojima M, et al. Increased nuclear factor- $\mathrm{kB}$ activation in human colorectal carcinoma and its correlation with tumor progression. Anticancer Res. 2004;24(2B):675-681.

9. Scartozzi M, et al. Nuclear factor-kB tumor expression predicts response and survival in irinotecan-refractory metastatic colorectal cancer treated with cetuximab-irinotecan therapy. J Clin Oncol. 2007;25(25):3930-3935.

10. Greten FR, et al. IKK $\beta$ links inflammation and tumorigenesis in a mouse model of colitis-associated cancer. Cell. 2004;118(3):285-296

11. Cusack JC, Liu R, Baldwin AS. Inducible chemoresistance to 7-ethyl-10-[4-(1-piperidino)-1-piperidino]-carbonyloxycamptothecin (CPT-11) in colorectal cancer cells and a xenograft model is overcome by inhibition of nuclear factor- $\mathrm{kB}$ activation. Cancer Res. 2000;60(9):2323-2330.

12. Gilmore TD, Herscovitch M. Inhibitors of NF-кB signaling: 785 and counting. Oncogene. 2006;25(51):6887-6899.

13. Erstad DJ, Cusack JC. Targeting the NF-кB pathway in cancer therapy. Surg Oncol Clin N Am. 2013;22(4):705-746

14. Prescott JA, Cook SJ. Targeting IKK $\beta$ in cancer: challenges and opportunities for the therapeutic utilisation of IKK $\beta$ inhibitors. Cells. 2018;7(9):E115.

15. Beg AA, Sha WC, Bronson RT, Ghosh S, Baltimore D. Embryonic lethality and liver degeneration in mice lacking the RelA 
component of NF-кB. Nature. 1995;376(6536):167-170.

16. Tanaka M, et al. Embryonic lethality, liver degeneration, and impaired NF-kB activation in IKK- $\beta$-deficient mice. Immunity. 1999;10(4):421-429

17. Jain A, Kaczanowska S, Davila E. IL-1 receptor-associated kinase signaling and its role in inflammation, cancer progression, and therapy resistance. Front Immunol. 2014;5:553.

18. Lim KH, Staudt LM. Toll-like receptor signaling. Cold Spring Harb Perspect Biol. 2013;5(1):a011247.

19. Ngo VN, et al. Oncogenically active MYD88 mutations in human lymphoma. Nature. 2011;470(7332):115-119.

20. Kelly PN, et al. Selective interleukin-1 receptor-associated kinase 4 inhibitors for the treatment of autoimmune disorders and lymphoid malignancy. J Exp Med. 2015;212(13):2189-2201.

21. Rhyasen GW, et al. Targeting IRAK1 as a therapeutic approach for myelodysplastic syndrome. Cancer Cell. 2013;24(1):90-104

22. Li Z, et al. Inhibition of IRAK1/4 sensitizes T cell acute lymphoblastic leukemia to chemotherapies. J Clin Invest. 2015;125(3):1081-1097.

23. Srivastava R, et al. Augmentation of therapeutic responses in melanoma by inhibition of IRAK-1,-4. Cancer Res. 2012;72(23):6209-6216.

24. Wee ZN, et al. IRAK1 is a therapeutic target that drives breast cancer metastasis and resistance to paclitaxel. Nat Commun. 2015;6:8746.

25. Adams AK, et al. IRAK1 is a novel DEK transcriptional target and is essential for head and neck cancer cell survival. Oncotarget. 2015;6(41):43395-43407.

26. Zhang D, et al. Constitutive IRAK4 activation underlies poor prognosis and chemoresistance in pancreatic ductal adenocarcinoma. Clin Cancer Res. 2017;23(7):1748-1759.

27. Zhang D, et al. Tumor-stroma IL1 $\beta$-IRAK4 feedforward circuitry drives tumor fibrosis, chemoresistance, and poor prognosis in pancreatic cancer. Cancer Res. 2018;78(7):1700-1712.

28. Singh A, et al. TAK1 inhibition promotes apoptosis in KRAS-dependent colon cancers. Cell. 2012;148(4):639-650.

29. Tang M, et al. TAK1 is required for the survival of hematopoietic cells and hepatocytes in mice. J Exp Med. 2008;205(7):1611-1619.

30. Cooper HS, et al. The role of mutant Apc in the development of dysplasia and cancer in the mouse model of dextran sulfate sodium-induced colitis. Gastroenterology. 2001;121(6):1407-1416.

31. Suzuki N, Saito T. IRAK-4 - a shared NF-кB activator in innate and acquired immunity. Trends Immunol. 2006;27(12):566-572.

32. Smith MA, et al. U2AF1 mutations induce oncogenic IRAK4 isoforms and activate innate immune pathways in myeloid malignancies. Nat Cell Biol. 2019;21(5):640-650.

33. Rhodes DR, et al. ONCOMINE: a cancer microarray database and integrated data-mining platform. Neoplasia. 2004;6(1):1-6.

34. Akagi J, et al. Therapeutic antitumor response after immunization with an admixture of recombinant vaccinia viruses expressing a modified MUC1 gene and the murine T-cell costimulatory molecule B7. J Immunother. 1997;20(1):38-47.

35. Lee KL, et al. Discovery of clinical candidate 1-\{[(2S,3S,4S)-3-ethyl-4-fluoro-5-oxopyrrolidin-2-yl]methoxy $\}$-7-methoxyisoquinoline-6-carboxamide (PF-06650833), a potent, selective inhibitor of interleukin-1 receptor associated kinase 4 (IRAK4), by fragment-based drug design. J Med Chem. 2017;60(13):5521-5542.

36. Kondo M, et al. Renoprotective effects of novel interleukin-1 receptor-associated kinase 4 inhibitor AS2444697 through anti-inflammatory action in 5/6 nephrectomized rats. Naunyn Schmiedebergs Arch Pharmacol. 2014;387(10):909-919.

37. Mercurio F, et al. IKK-1 and IKK-2: cytokine-activated IkappaB kinases essential for NF-кB activation. Science. 1997;278(5339):860-866.

38. McCool KW, Miyamoto S. DNA damage-dependent NF-kB activation: NEMO turns nuclear signaling inside out. Immunol Rev 2012;246(1):311-326.

39. Durand JK, Baldwin AS. Targeting IKK and NF-кB for therapy. Adv Protein Chem Struct Biol. 2017;107:77-115.

40. Lim KH, Staudt LM. Toll-like receptor signaling. Cold Spring Harb Perspect Biol. 2013;5(1):a011247.

41. Chou TC. Drug combination studies and their synergy quantification using the Chou-Talalay method. Cancer Res. 2010;70(2):440-446.

42. Jiang H, et al. Targeting focal adhesion kinase renders pancreatic cancers responsive to checkpoint immunotherapy. Nat Med. 2016;22(8):851-860

43. Suzuki N, et al. Severe impairment of interleukin-1 and Toll-like receptor signalling in mice lacking IRAK-4. Nature. 2002;416(6882):750-756.

44. $\mathrm{Ku} \mathrm{CL}$, et al. Selective predisposition to bacterial infections in IRAK-4-deficient children: IRAK-4-dependent TLRs are otherwise redundant in protective immunity. J Exp Med. 2007;204(10):2407-2422.

45. Picard C, et al. Clinical features and outcome of patients with IRAK-4 and MyD88 deficiency. Medicine (Baltimore). 2010;89(6):403-425

46. Zackular JP, Baxter NT, Chen GY, Schloss PD. Manipulation of the gut microbiota reveals role in colon tumorigenesis. $m$ Sphere 2016;1(1):e00001-15.

47. Zackular JP, et al. The gut microbiome modulates colon tumorigenesis. MBio. 2013;4(6):e00692-e00613.

48. Wong SH, et al. Gavage of fecal samples from patients with colorectal cancer promotes intestinal carcinogenesis in germ-free and conventional mice. Gastroenterology. 2017;153(6):1621-1633.e6.

49. Salcedo R, Cataisson C, Hasan U, Yuspa SH, Trinchieri G. MyD88 and its divergent toll in carcinogenesis. Trends Immunol. 2013;34(8):379-389.

50. Scheeren FA, et al. A cell-intrinsic role for TLR2-MYD88 in intestinal and breast epithelia and oncogenesis. Nat Cell Biol. 2014;16(12):1238-1248.

51. Rakoff-Nahoum S, Medzhitov R. Regulation of spontaneous intestinal tumorigenesis through the adaptor protein MyD88. Science. 2007;317(5834):124-127.

52. Lee SH, et al. ERK activation drives intestinal tumorigenesis in Apc(min/+) mice. Nat Med. 2010;16(6):665-670.

53. Salcedo R, et al. MyD88-mediated signaling prevents development of adenocarcinomas of the colon: role of interleukin 18 . J Exp Med. 2010;207(8):1625-1636.

54. Araki A, et al. MyD88-deficient mice develop severe intestinal inflammation in dextran sodium sulfate colitis. J Gastroenterol. 2005;40(1):16-23. 
55. Schiechl G, et al. Tumor development in murine ulcerative colitis depends on MyD88 signaling of colonic F4/80+CD11b(high) Gr1(low) macrophages. J Clin Invest. 2011;121(5):1692-1708.

56. Xie L, et al. Targeting of MyD88 homodimerization by novel synthetic inhibitor TJ-M2010-5 in preventing colitis-associated colorectal cancer. J Natl Cancer Inst. 2016;108(4):djv364.

57. Wang EL, et al. High expression of Toll-like receptor 4/myeloid differentiation factor 88 signals correlates with poor prognosis in colorectal cancer. Br J Cancer. 2010;102(5):908-915.

58. Auclin E, et al. Subgroups and prognostication in stage III colon cancer: future perspectives for adjuvant therapy. Ann Oncol. 2017;28(5):958-968.

59. Dienstmann R, Salazar R, Tabernero J. Personalizing colon cancer adjuvant therapy: selecting optimal treatments for individual patients. J Clin Oncol. 2015;33(16):1787-1796.

60. Ding Q, et al. APOBEC3G promotes liver metastasis in an orthotopic mouse model of colorectal cancer and predicts human hepatic metastasis. J Clin Invest. 2011;121(11):4526-4536.

61. Nalbantoglu I, Blanc V, Davidson NO. Characterization of colorectal cancer development in Apc (min/+) mice. Methods Mol Biol. 2016;1422:309-327.

62. Cooper HS, Murthy SN, Shah RS, Sedergran DJ. Clinicopathologic study of dextran sulfate sodium experimental murine colitis. Lab Invest. 1993;69(2):238-249.

63. Cerami E, et al. The cBio cancer genomics portal: an open platform for exploring multidimensional cancer genomics data. Cancer Discov. 2012;2(5):401-404. 